\title{
Basic Quantum Magnetic Interaction Based on Detailed Gauss Meter Measurements
}

\author{
Bhekuzulu Khumalo \\ Private Researcher, Toronto, Canada \\ Email: bhekuzulu.khumalo@gmail.com
}

How to cite this paper: Khumalo, B. (2022) Basic Quantum Magnetic Interaction Based on Detailed Gauss Meter Measurements. Open Journal of Microphysics, 12, 1-30.

https://doi.org/10.4236/ojm.2022.121001

Received: November 1, 2021

Accepted: December 21, 2021

Published: December 24, 2021

Copyright $\odot 2022$ by author(s) and Scientific Research Publishing Inc. This work is licensed under the Creative Commons Attribution International License (CC BY 4.0).

http://creativecommons.org/licenses/by/4.0/ (c) (i) Open Access

\begin{abstract}
Understanding that the magnetic phenomenon is both a particle, khumalon, that organizes itself into a wave formation to travel through a medium, this paper shows proof of this in detail. It can only be doing this because it is traveling through some medium. This paper is about observing relationships that are a result of different polarized magnetic phenomenon forced into relationships. The magnet shows it does not behave differently from other particles; when they have relationships with their "anti" particle, annihilation takes place. Two magnets will always be like a collider, but because the magnetic phenomenon is already unstable and of low energy allows us to see things otherwise difficult with particles of higher energy. Isaac Newton is truly needed in explaining this phenomenon. The magnetic phenomenon adheres to the first two laws of everything. All data can be found on figshare.com, to share.
\end{abstract}

\section{Keywords}

Isaac Newton, Khumalon, Line of Silence/Annihilation, Magnetic Wave, Magnetism, Particle Annihilation, Quantum Magnetism

\section{Introduction}

Having confirmed that the magnetic phenomenon must be a discrete phenomenon that organizes itself into a wave in the papers "[1] Magnetism: Insights from the Thomas Young Experiment" and "[2] Magnetism: Further Proof of Wave Particle Duality" that were based on experiments carried out by the author of this paper.

No literature review as such is possible, nobody knew magnetism organizes itself into a wave, it will be illogical to include what one never did as some inspiration, otherwise they would have done the experiments and told us magnetism 
organizes into a wave.

As we see the magnetic phenomenon organizes itself into a wave formation, it can only mean:

- It must take this formation, as it always eventually ends up in this formation.

- Having to take this formation from no formation means this is a law of its existence, it is the way it travels most efficiently. Being the way, it travels most efficiently, it must be travelling through a medium of some sort, this medium could be dark matter/energy or the ether, it does not matter, what matters is, it is traveling through a medium.

- It must be interacting with this medium hence the need to take this formation.

- To get to this formation from chaos can only be determined by an algorithm, if it is a quantum phenomenon, it must adhere to Schrodinger's equations as it travels. Because this [3] algorithm determines relationships in basic particles, at its most complex it is controlling existence, hence ability to successfully use mathematics to show relationships in science. This is merely because it determines the formation a khumalon will take as it travels, a khumalon being the particle associated with magnetism. To deny existence of a khumalon is to deny experimentation.

We now understand that the phenomenon position can be determined by Schrodinger's equations; however this equation must be limited by:

$$
M_{p h}=a \mathrm{e}^{-K_{e} P_{e} c}
$$

where:

$$
\begin{aligned}
& M_{p h}=\text { magnetic phenomenon } \\
& a=\text { magnetic force at face } \\
& \text { e = natural exponential function } \\
& K_{e}=\text { kinetic energy } \\
& P_{e}=\text { potential energy/amount of matter } \\
& c=\text { speed of light }
\end{aligned}
$$

This is because the magnetic phenomenon decreases at an exponential rate. The only plausible reason for this is that the magnetic phenomenon, the khumalon is a highly unstable particle. We see this behaviour in particle colliders, a huge variety of material that is unstable; the magnet is one of them. Within 2 jiffies more than $85 \%$ of this stuff has disappeared, as unstable as anything being produced in any collider.

The khumalon allows us to both understand unstable and stable behaviour in quantum mechanics, because though most of the magnetic phenomenon will disappear in 3 jiffies, some of the stuff can exist for more than a second.

The earths [4] magnetic field ranges from about 65,000 kilometers $(40,000$ miles) - 600,000 kilometers (370,000 miles). The [5] speed of light is around 300,000 kilometers per second. This means we are detecting a magnetic field that has this phenomenon lasting for between 0.22 seconds and 2 seconds. Obviously with a thorough study we will learn more because we will have to take into con- 
sideration the seemingly considerable interaction between the earths magnetic field and cosmic information from the sun and deep inside the universe.

\section{Aims}

The aims of this paper are to move on from merely identifying the magnetic phenomenon as a particle and has wave like formation as it travels like all other quantum phenomena.

How do we explain magnetic interaction in terms of particles and waves, in terms of quantum theory? The aims of this paper are just that, to discuss basic magnetic interaction in terms of quantum phenomenon, in terms of this phenomenon's true nature. What are the relationships that are taking place as the phenomenon interacts, these are the basic questions that must be answered?

Quantum magnetic interaction could never be understood without first understanding what magnetism is, it is a quantum phenomenon that organizes itself into a wave. Nobody could understand it because solving what magnetism is was always an experimental problem, not a mathematical problem. Drawing up equations was never going to tell us that magnetism organizes into a wave, one had to measure. It is from these measurements we derive the equations for the interactions, not the other way round.

Magnetism is something unique. "[6] Quantum correlations have a non-electric and non-magnetic nature and take place independent of the distance between the interacting quantum entities." Photons for example are not electrons, electrons are electrons, photons will have own unique characteristics. Photons and electrons are not magnetic and will not have the characteristics of a magnetic wave. Though magnetism is associated with charge, it is not the charge, an electron emits khumalons, but is not a khumalon. Everything is unique in all the similarities.

\section{The Experiments}

There are three experiments that were undertaken for this paper. There is a control experiment, experiment 1 . There is then an experiment where we test relationships between magnets with opposing poles, this is experiment 2 . The last experiment is when we compare interaction and relationships between magnets with similar poles.

Though talking of 3 experiments, they can all be considered as one experiment in three parts. All the data for these experiments can be found in the appendix.

The size of the magnets used in the experiment is ceramic and is of the dimensions set out in Figure 1.

\subsection{Experiment 1}

This is considered the control experiment. It is to give us a base, something to compare with. This experiment involved measuring $80 \mathrm{~mm}$ away from a magnet as depicted in Figure 112 time starting from the face of the magnet. The mea- 
surement grid is given below, each line being $0.5 \mathrm{~cm}, 5 \mathrm{~mm}$ apart as depicted in Figure 2.

As the experiment involved moving away from the source of the magnetic phenomenon it means we have 12 essentially independent measurements moving away. This is a different technique than what was used in the experiments for the papers "Magnetism: Insights from the Thomas Young Experiment. In these experiments the magnetic field was measured across the face of the source of the magnetic phenomenon.

Figure 3 shows the results of experiment 1 graphically, the data can be seen in appendix A.

As can be seen from Figure 3, we have some negative numbers, these represent magnetic phenomenon that is south polarized. This is the base experiment; the rest of the experiments are about what happens to these base results. This should be no surprise as such, but a surprise nonetheless. When we look at where this opposite polarized phenomenon is at, lines 1 and 12 , we see it is beyond the boundaries of the magnetic face. Then the next 2 lowest are at the boundaries of the magnetic face, lines 2 and 11.

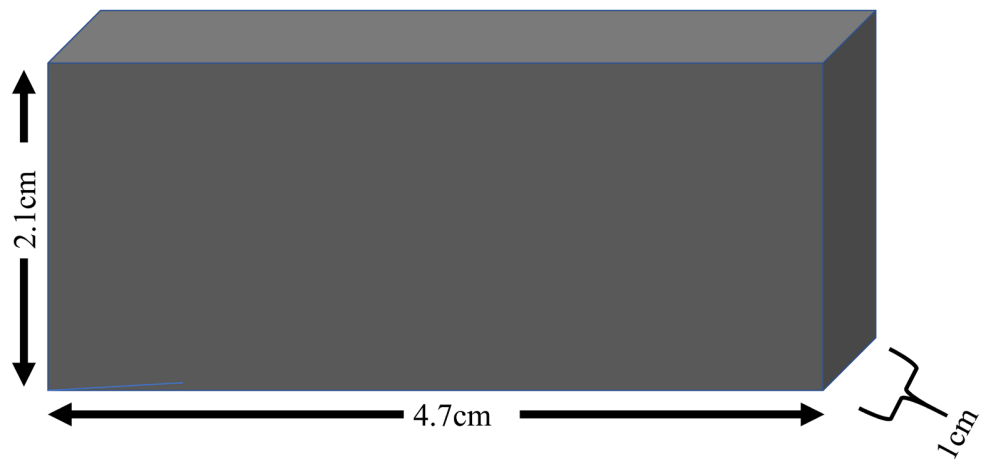

Figure 1. Dimension of magnets used.

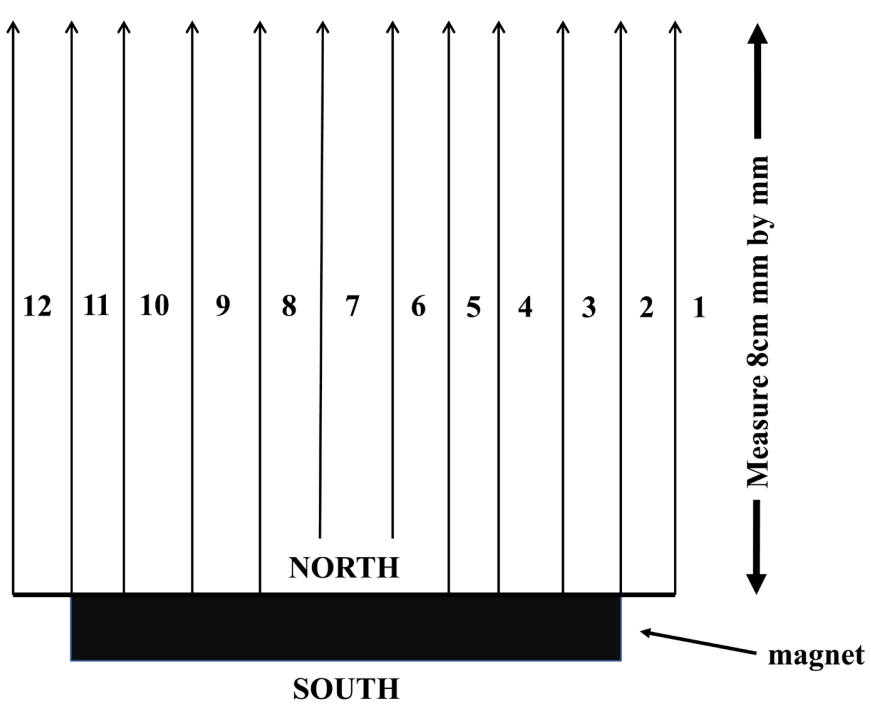

Figure 2. Base experiment. 


\subsubsection{The Obvious}

1) Magnetism is strongest at poles as can be seen in Figure 4, but as one moves away it organizes into a wave and strongest at center.

2) Equation (1) is a general equation limiting the amount of phenomenon per period or distance travelled. As we can see though most of the phenomenon has the same pattern graphically as can be seen from figure however a, the phenomenon at the face is always different, substantially. $K_{e}$ will be different for each phenomenon, however $P_{e}$ will be the same for all, that is what defines the phenomenon allowing us to categorize it as magnetic.

3) That the experiment involved measuring moving away from the face and it

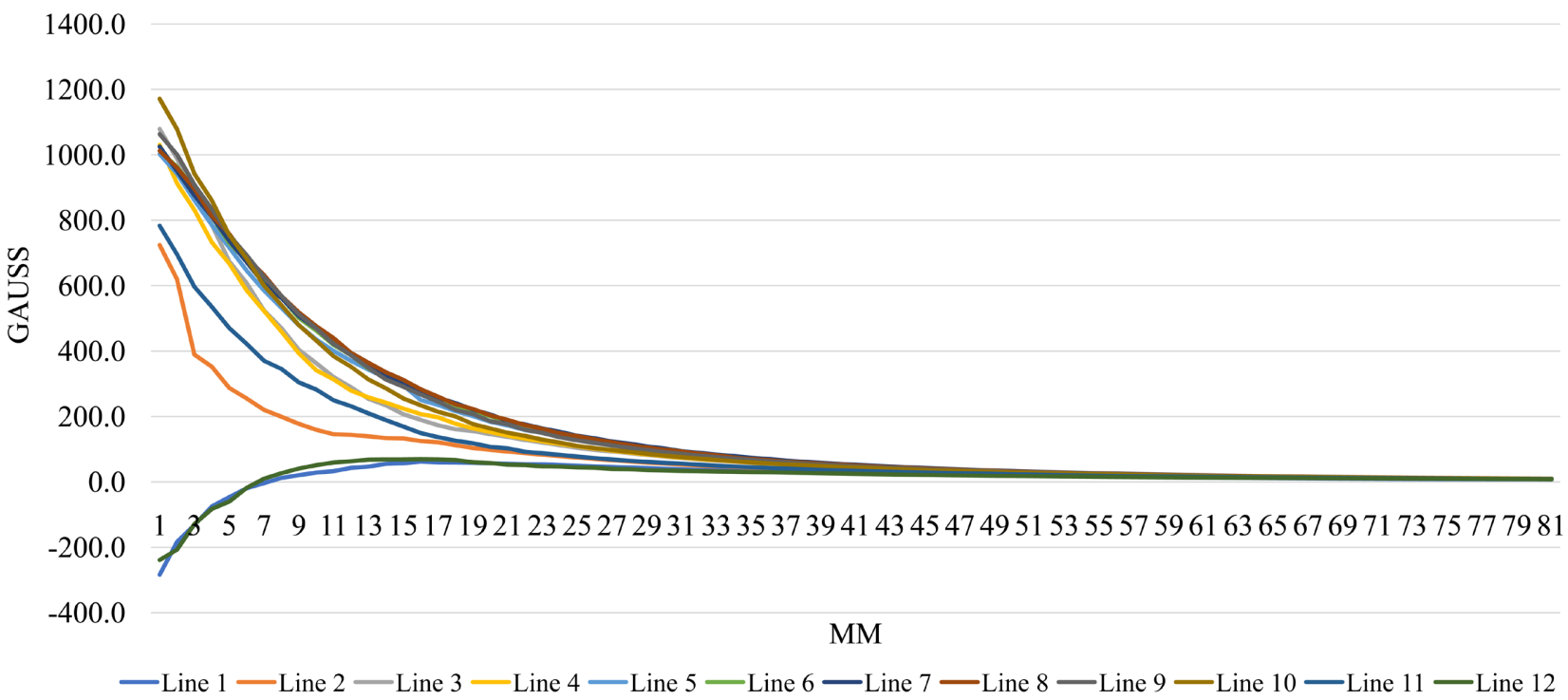

Figure 3. Experiment 1.

1200.0

1150.0

1100.0

忩 1050.0

1000.0

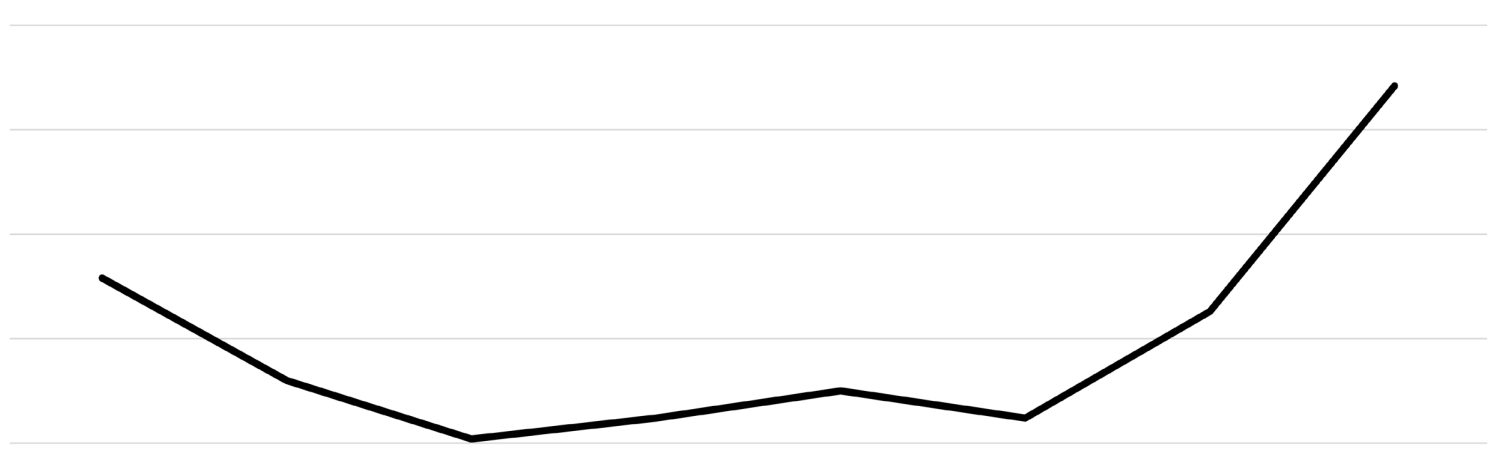

950.0

900.0

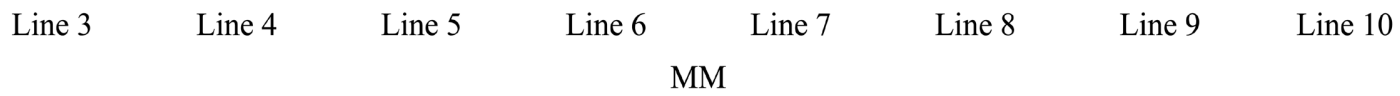

Figure 4. At face. 
confirms the patterns that where received moving across the face not only gives us greater detail, $1 \mathrm{~mm}$ away, $2 \mathrm{~mm}$ away and the such, but the phenomenon exhibits same pattern of wave particle is a testament to the accuracy of the home made measuring instrument. Not so much the gauss meter, but the transportation system.

\subsubsection{First Half a Jiffy}

A jiffy is the time it takes for the speed of light to travel $1 \mathrm{~cm}$, in a vacuum, but even outside a vacuum, speed of light is amazingly fast relative to speeds that we can reach. Not forgetting that in one second time travels 300,000 kilometers. When one talks about half a jiffy, one is talking about the first $5 \mathrm{~mm}$ of this phenomenon.

Figure 5(a) shows the first $5 \mathrm{~mm}$ for all measurements.

There is still a lot of differences no real wave structure as one moves from one millimetre to the next. This is more easily seen if we just take a glance of what is happening in front of the face from lines 3 to 10 as depicted in Figure 5(b).

One can compare Figure 5(c), the phenomenon across the face at $5 \mathrm{~mm}$ and Figure 4, this is the difference of the pattern at the face and after traveling for two fifths of a jiffy, not forgetting that measurement 1 is the face.

Looking at the strengths of magnetic phenomenon at $0.5 \mathrm{~cm}$ is what was done with above mentioned papers, but here we can even compare at $2 \mathrm{~mm}$, at 0.1 of a jiffy as depicted in Figure 5(d).

The detail of this experiment allows us to look at the phenomenon at $2 \mathrm{~mm}$, there is a significant difference between $2 \mathrm{~mm}$ and $5 \mathrm{~mm}$ in the pattern of how the phenomenon is moving. It is organizing itself.

We know the eventual formation will be a wave like formation, but we can see the phenomenon organizing itself.

\section{Staggering Number of Calculations}

Taking the Planck Length as the "smallest" length possible, a khumalon can be said to approximate a Planck length. $1 \mathrm{~cm}$ is equal to ${ }^{7} 6.1879273537329 \mathrm{E}+32$ Planck length, that is how many khumalons would be in one centimeter. That means in $5 \mathrm{~mm}$ there would be $3.09 \mathrm{E}+32$ of this phenomenon.

In our scenario, given magnet let us say $4.5 \mathrm{~cm}$, it means at any time $2.78 \mathrm{E}+33$ of this phenomenon is leaving the magnet. In $5 \mathrm{~mm}$, half a jiffy there is potentially $2.78 \mathrm{E}+33$ multiplied by $3.09 \mathrm{E}+32$ positions that can be taken, potentially $8.62 \mathrm{E}+65$ calculations in half a jiffy, far more powerful even than the most powerful visualized quantum computer, there is just no comparison.

The above paragraph could be considered an exaggeration because we must consider the idea of sequencing and that space is occupied by the same phenomenon. The potential of calculations could be even $1 \%$ of that in half a jiffy, that is $8.62 \mathrm{E}+63$ calculations in a time so fast we cannot comprehend. When one talks of a second, the number of calculations are astronomical, by far, the most superior model to mimic we will ever find as humans for our own computers, the magnetic phenomenon organizing itself into a wave. 


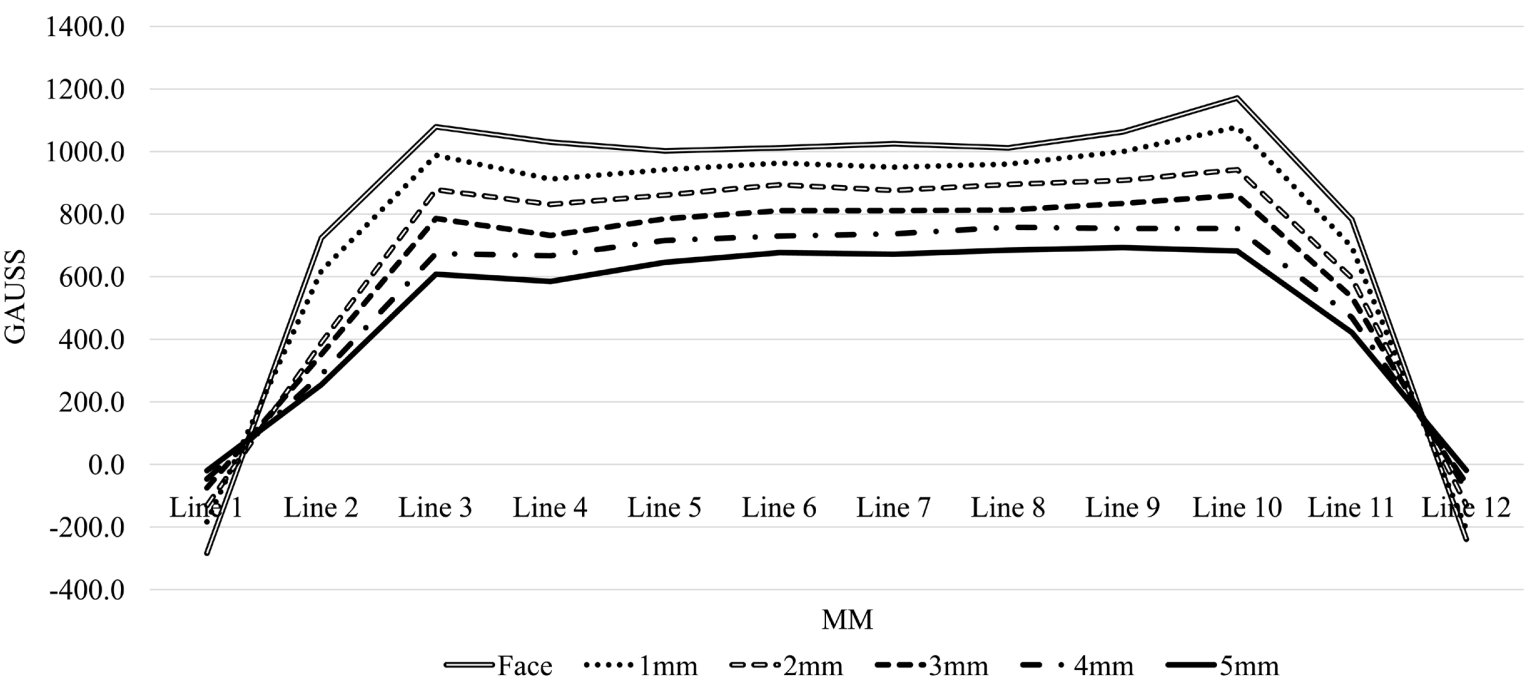

(a)

1400.0

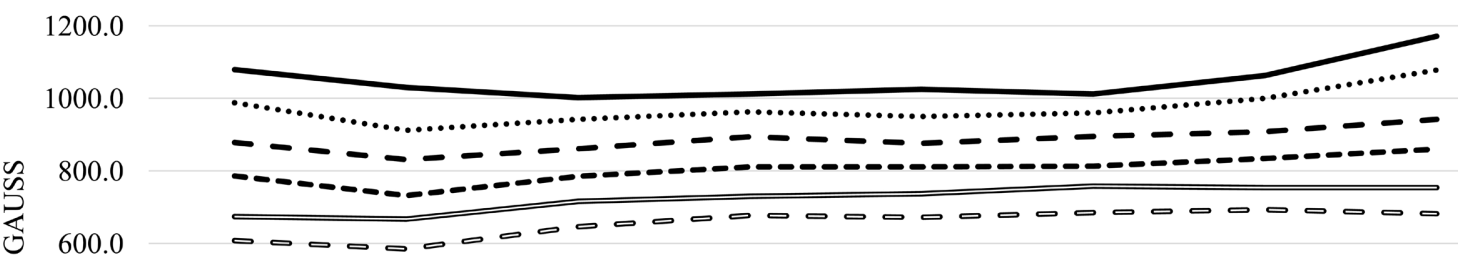

400.0

200.0

0.0

$$
\begin{aligned}
& \begin{array}{llllllll}
\text { Line } 3 & \text { Line } 4 & \text { Line } 5 & \text { Line } 6 & \text { Line } 7 & \text { Line } 8 & \text { Line } 9 & \text { Line } 10
\end{array} \\
& \text {-Face } \cdots \cdot 1 \mathrm{~mm} \quad-\quad-2 \mathrm{~mm} \quad--\cdot 3 \mathrm{~mm} \quad 4 \mathrm{~mm} \quad-\quad-5 \mathrm{~mm}
\end{aligned}
$$

(b)

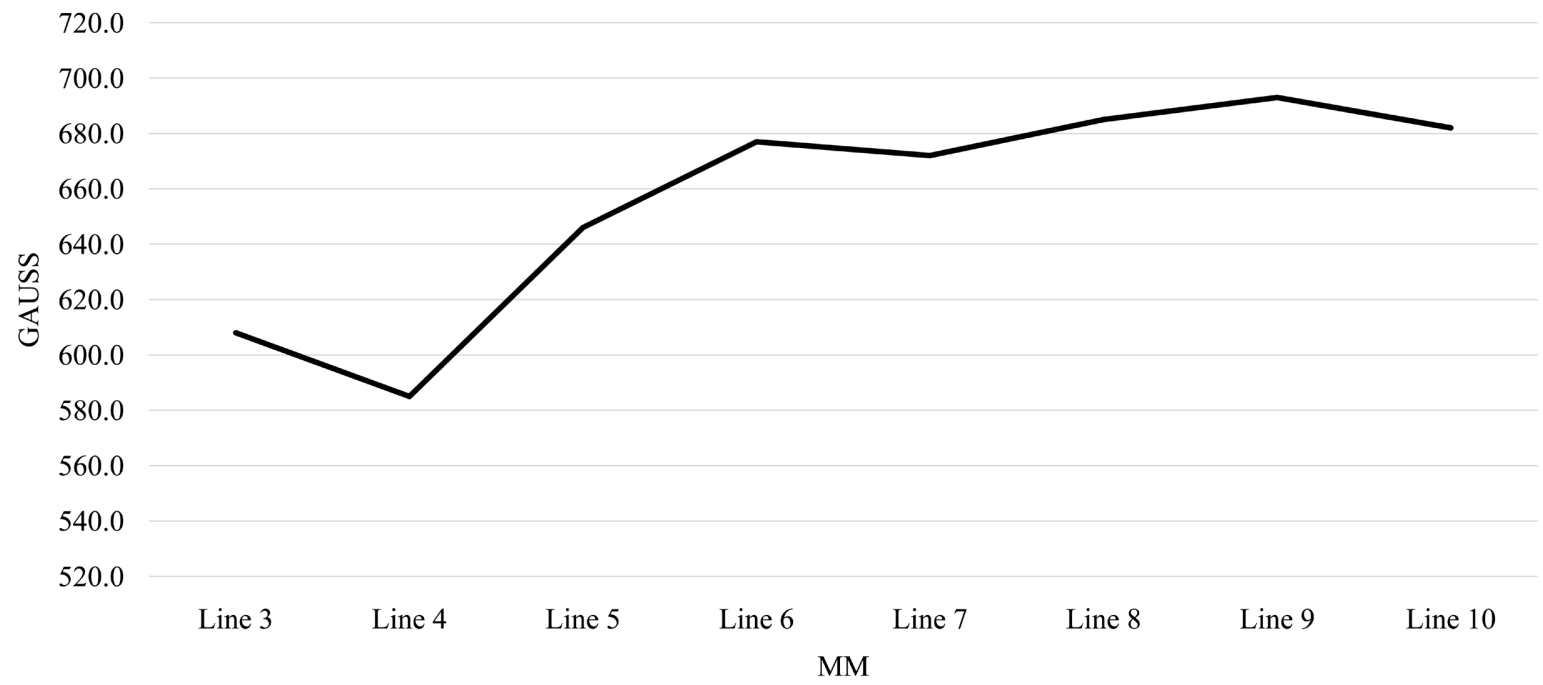

(c) 


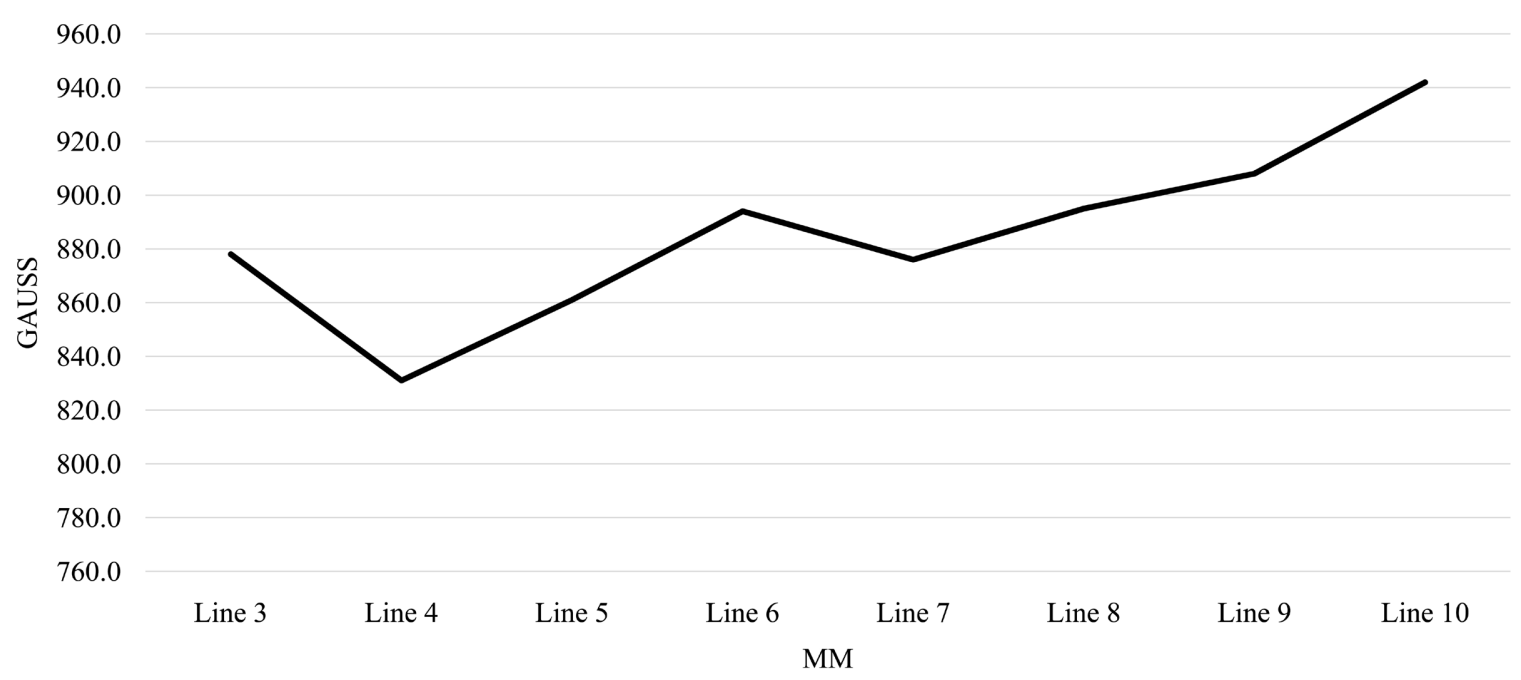

(d)

Figure 5. (a) First half a jiffy; (b) First half a jiffy line 3 - 10; (c) $5 \mathrm{~mm}$ from face; (d) $2 \mathrm{~mm}$ from face.

At half a jiffy this thing is still very disorganized but organizing itself. One could easily then conclude that it is about potential, there is potential that the magnetic field will be there, it is a field of potential. That would be wrong, we see it organizing itself. At much smaller levels an observer could very well be confused. The thing has a position but moves at the speed of light around an atom, well it could be anywhere. But we see a phenomenon is somewhere when not constrained in a relationship, it will organize itself for travel, in the most impressive way.

\subsubsection{Lines 14 - 20}

Lines 14 - 20 represent the phenomenon after $1.3-1.9$ jiffies, considering that the first measurement is at the face. The graphical representation can be seen in Figure 6(a).

We can take out line 1,2,11, and 12 and just consider the lines dealing with the face, 3 - 10 as in Figure 6(b).

The wave pattern established in after Line 18 is what is the pattern for the rest of the measurements except for 3 at the trail end but it re-established, tiny little differences when Line 8 is similar or just measured slightly higher, a 13 vs a 12.9 , a 10.7 vs a 10.6 .

Incidentally at measurement 16 , both lines 1 and 4 reach their maximum for North polarized magnetic phenomenon, having both started as South polarized. A testament to a high degree of accuracy of the tools used for collecting the data.

\subsubsection{0 - $43 \mathrm{~mm}$}

This pattern can be seen in Figure 7(a). As can be seen in Figure 7(a), the wave pattern is incorporating lines $1,2,11$, and 12 .

Though we can see that all the phenomena are being incorporated into a single wave, it would be interesting to know what is still happening between lines 3 - 10. We can see the shape in Figure 7(b). 


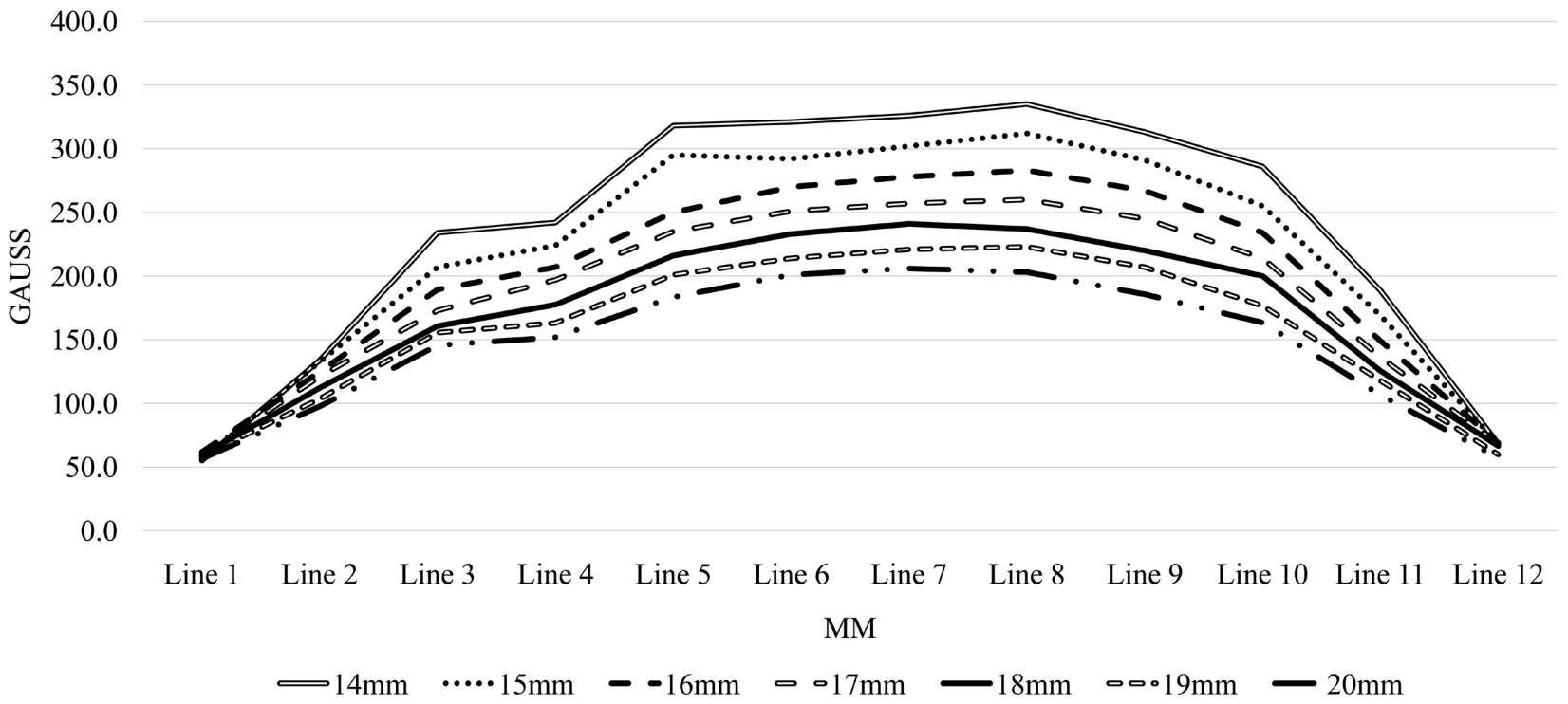

(a)

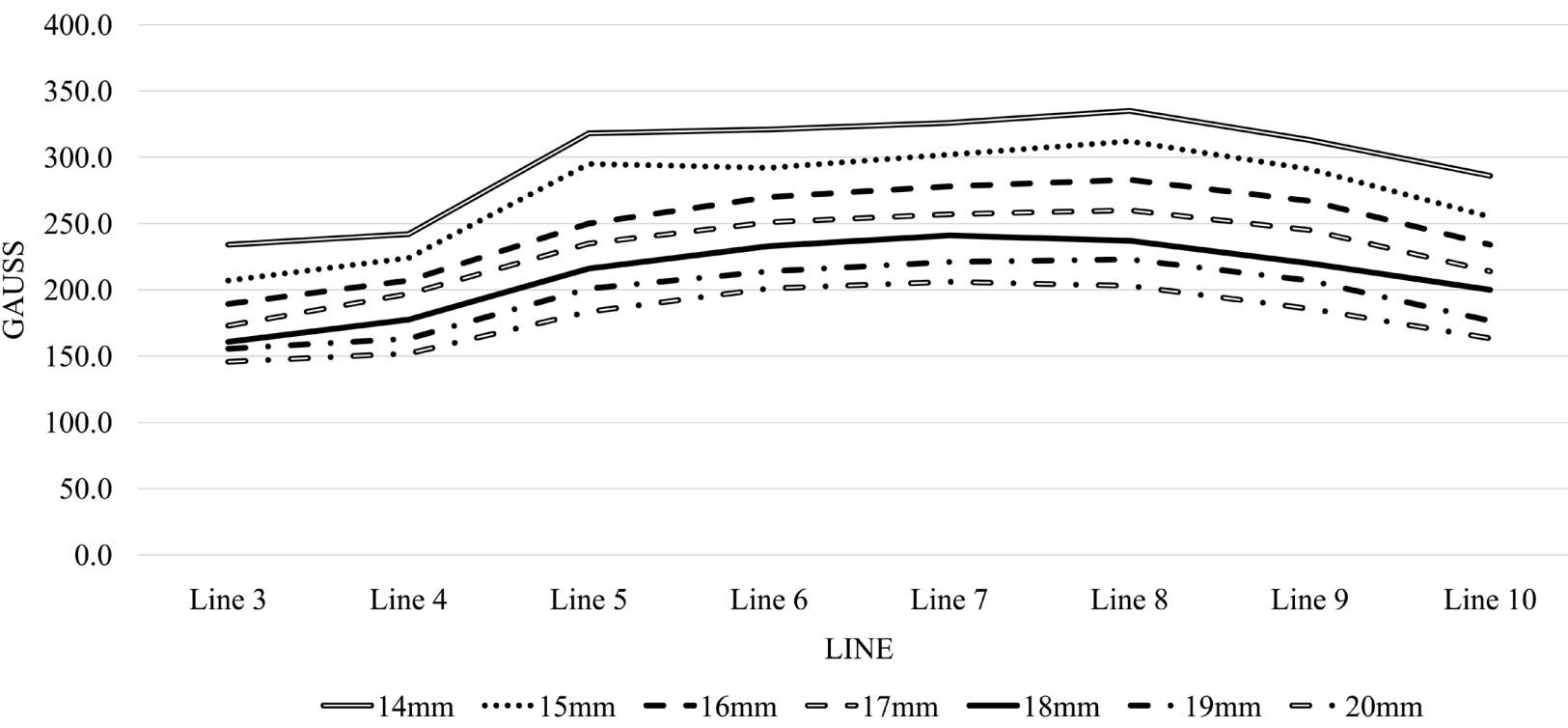

(b)

Figure 6. (a) $14 \mathrm{~mm}$ - $20 \mathrm{~mm}$; (b) Lines 3 - 1014 - $22 \mathrm{~mm}$.

\subsubsection{Lines 75 - 81}

Figure 8 shows what is happening at Lines $75-81$, at the end of our measurements.

What experiment 1 has shown is it confirms that this is a discrete phenomenon that takes a wave pattern It takes this wave pattern because it must be going through a medium, why not remain in that chaotic state as in the first half a jiffy, why organize?

It was not the purpose of experiment 1 to further prove the existence of discrete magnetic phenomenon because of its behaviour. It was meant to be a base for the next experiments, but the data revealed in greater detail this wave particle behaviour. 


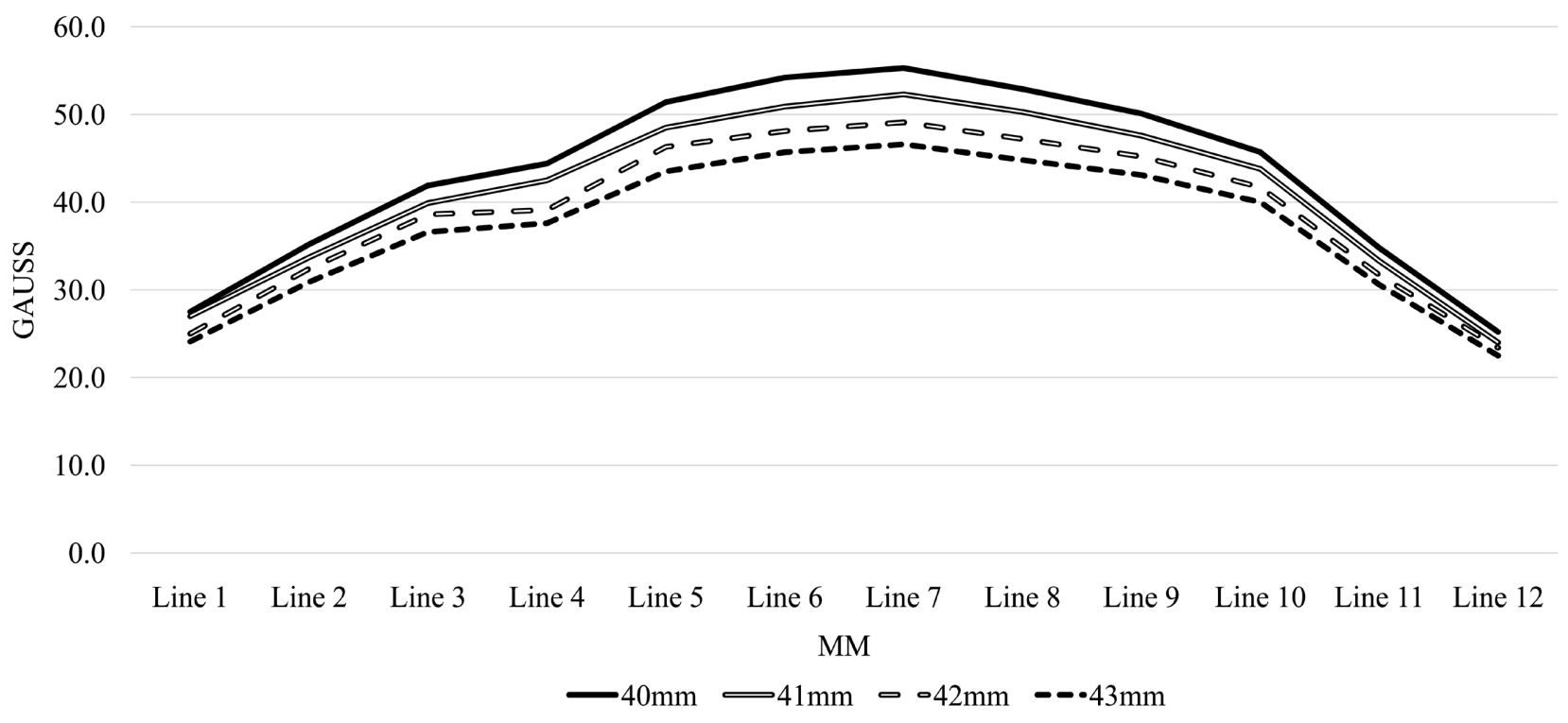

(a)

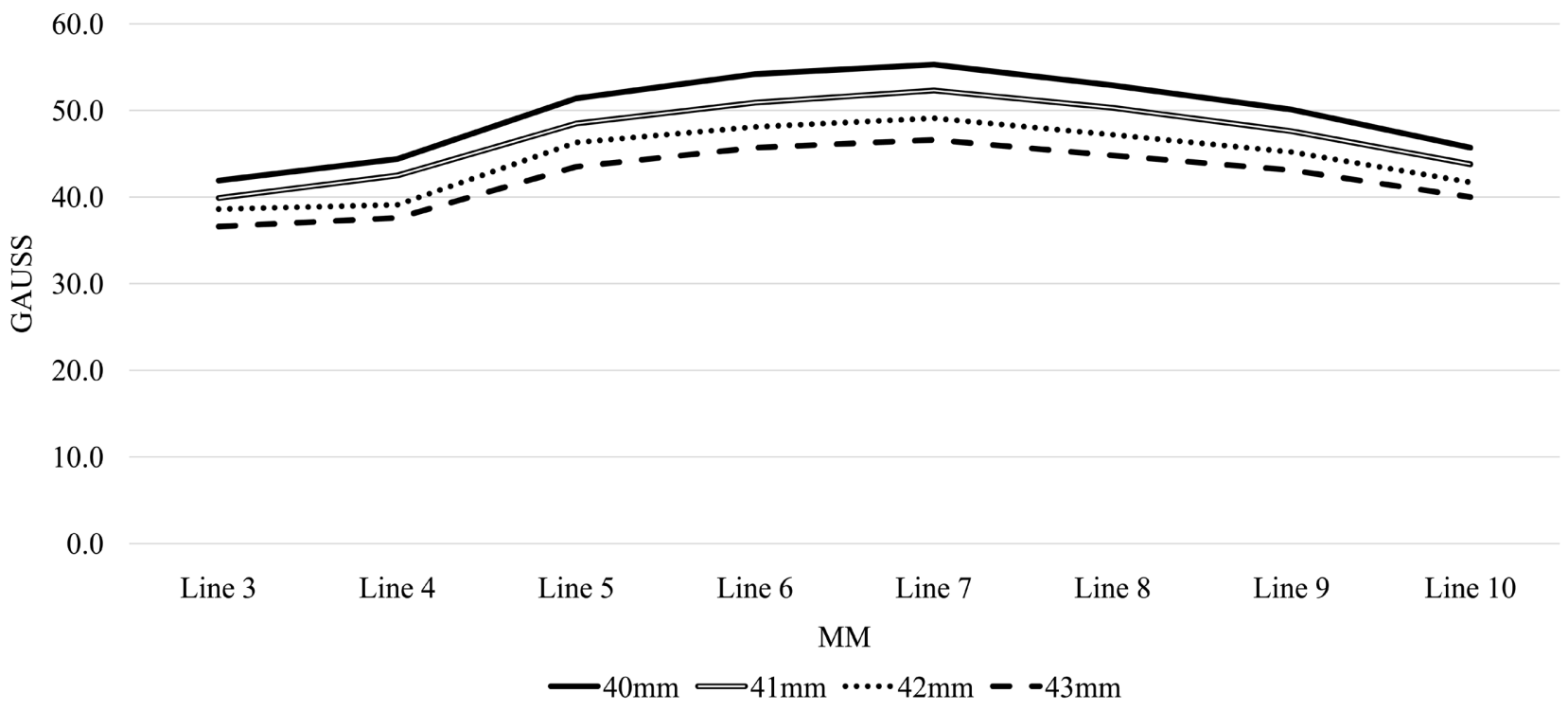

(b)

Figure 7. (a) $40 \mathrm{~mm}$ - $43 \mathrm{~mm}$; (b) Lines 3 - 1040 - $43 \mathrm{~mm}$.

Appendix D has 4 interesting tables, if we can ever understand what is going on, easily, the best mimic of reality we can do with our computers. The phenomenon is constantly adjusting itself as it moves, not just as in Equation (1), but the position of the phenomenon as we can see the percentage lost at each line, each mm every time.

These losses are never constant such that it means there is always constant adjustment, first to get to the wave formation and then to maintain it as it moves through this medium. We see this unique feature because the magnetic phenomenon is unstable, thus it will always be adjusting itself to maintain a wave structure. 


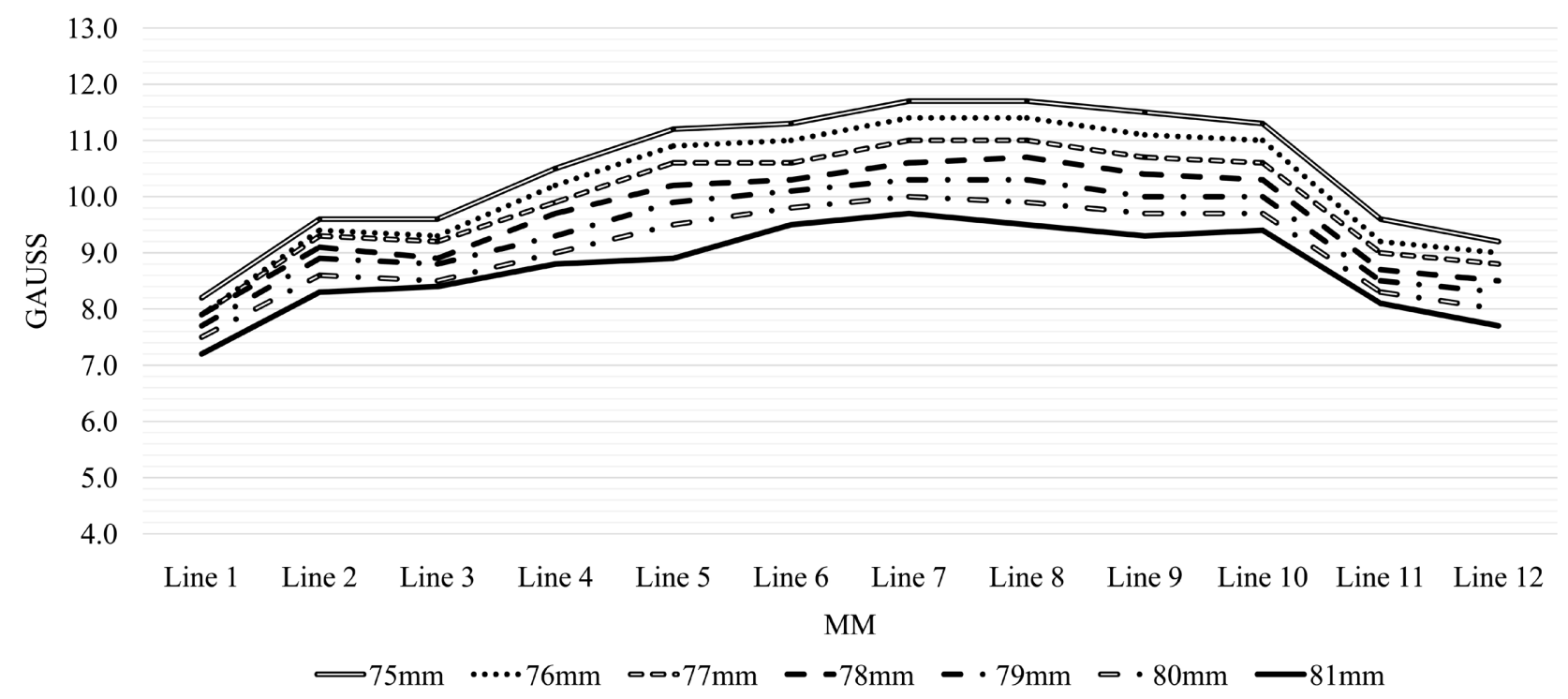

Figure 8. $75-80 \mathrm{~mm}$.

\subsection{Experiment 2}

This experiment was about testing what happens to the base experiment given another magnet to interact with the base magnet. The same 12 lines were measured, the data for this experiment can be found in appendix B.

The design of the experiment can be seen in Figure 9. The design was such that we wanted to see what happens when we put these magnets at a $90^{\circ}$ angle rather than them facing each other. The reasoning was that at this point, at this level, it is understood that magnetism is a particle called a khumalon, as the magnetic phenomenon moves away it adjusts itself.

What the scientist wanted to see with this experiment was what happens when we force two magnetic fields of opposing polarity to meet at $90^{\circ}$, given that these are particles we are dealing with, how will these particles interact, these differently polarized khumalons.

Figure $10(a)$ is from the data and makes a graphical visual presentation of that data. Figure 10(a) gives a lot of information, however, to get another visual view, graphical view, we can look at the phenomenon after 2.5 jiffies. To get rid of the huge distortions before that.

One can see from Figure 10(b) that there is significant interaction between the North and South Poles. The negative being the amount of South Pole magnetic phenomenon there is.

The experiment was designed such that we see what happens to the magnetic phenomenon away from original magnet and nothing else. We can see that a significant amount of south polarized magnetic phenomenon

\section{What Is Happening}

It was deliberate the $90^{\circ}$ angle interaction, but what is happening, then we can see the cause of what is called attraction (Figure 11). 


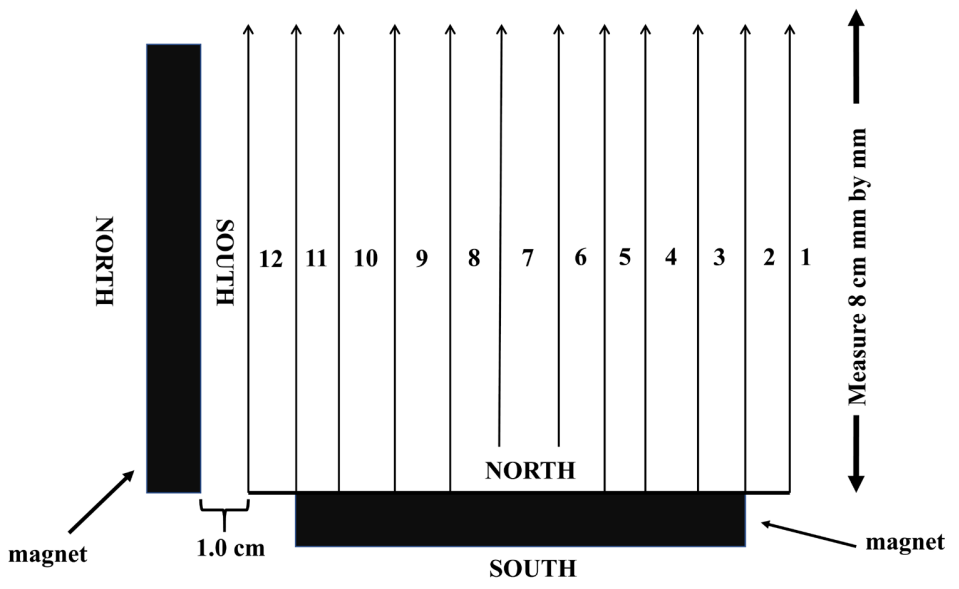

Figure 9. North south $90^{\circ}$ experiment 2.

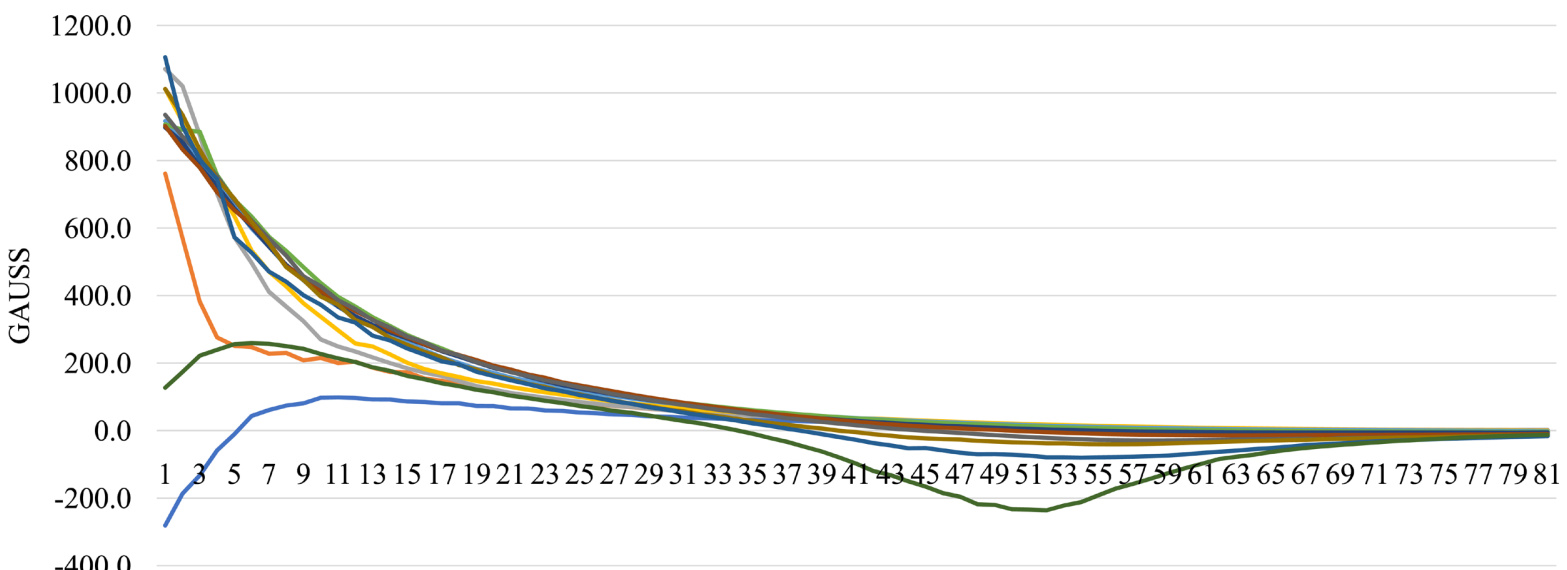

MM

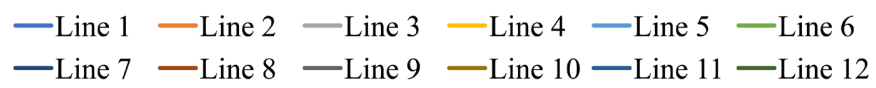

(a)

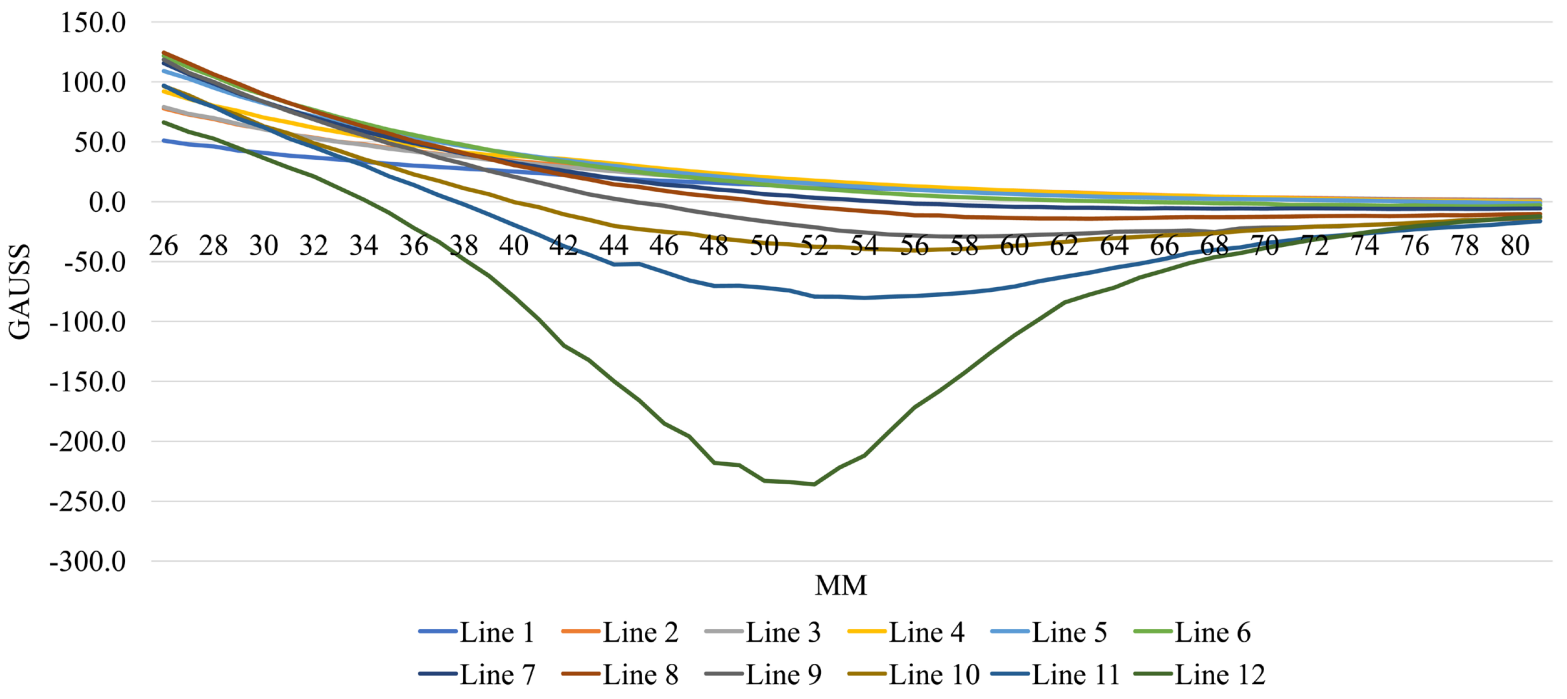

(b)

Figure 10. (a) Experiment 2; (b) Experiment 226 - 81. 


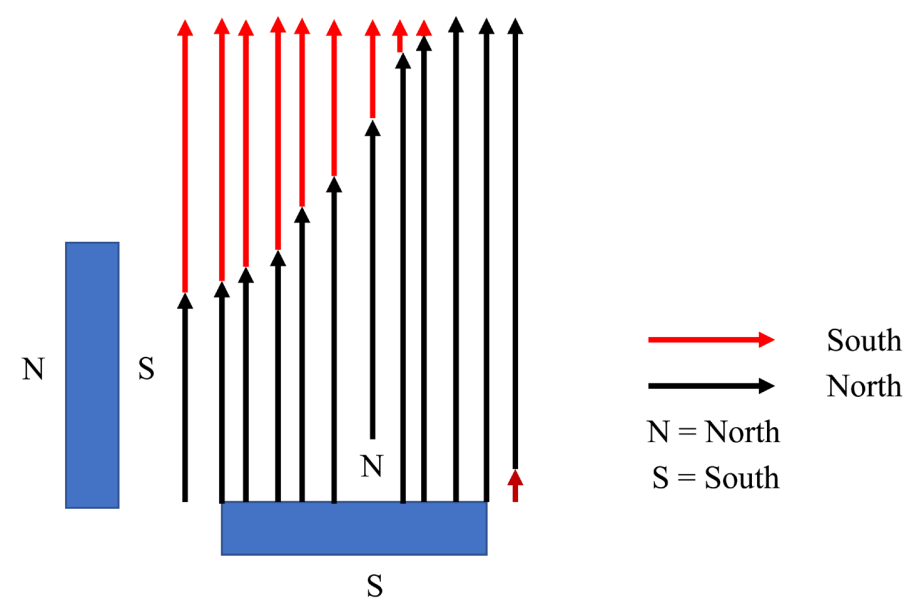

Figure 11. North south magnetic interaction.

Figure 11 shows what is happening directionally due to the addition of another magnet with opposite pole. There is a point after the interaction is strong enough when what is being measured in the opposite pole that the base experiment, experiment 1 .

It is worth noting that though line 1 starts with opposite pole as in base experiment, line 12 does not. Line 12 only becomes south pole directionally after the $35^{\text {th }}$ measurement. Figure 12 shows what is happening when the directional measurement changes from North to South.

What can be immediately known from Figure 12 is that the strength of the magnetic phenomenon increases away from the line of "annihilation" be it the north or the south polarizes phenomenon, khumalon.

These are polar opposites, they are annihilating each other, causing a "magnetic vacuum" It is important to understand that we are measuring the effects on the base experiment. Clearly after the line of annihilation we can see that the phenomenon is south polarized instead of North.

When we look at the data, we see that the North polarized phenomenon declines very rapidly as compared to base experiment. This is because the phenomenon is annihilating itself. This creates a vacuum, and as nature "abhors" a vacuum, to close this vacuum it seems like attraction. The magnets will smash into each other. It is not attraction.

Given that we have $\mathrm{K}^{\mathrm{n}}$ and $\mathrm{K}^{\mathrm{s}}$ opposite polarized magnetic phenomenon, khumalon polarized north, $\mathrm{K}^{\mathrm{n}}$ and khumalon polarized south $\mathrm{K}^{\mathrm{s}}$. What is truly happening. That opposite polarized phenomenon can annihilate each other means they can occupy the same space. It is this reality of occupying the same space that causes annihilation, no 2 similar things can occupy the same space, $\mathrm{K}^{\mathrm{n}}$ and $\mathrm{K}^{\mathrm{s}}$ will be similar in every essence except their polarization. It is this occupying of the same space, what was thought of as impossible, that leads to the annihilation. There is no intention on the part of the particles to occupy the same space, placing magnets like that is interference, it would never happen without the hand of man. 


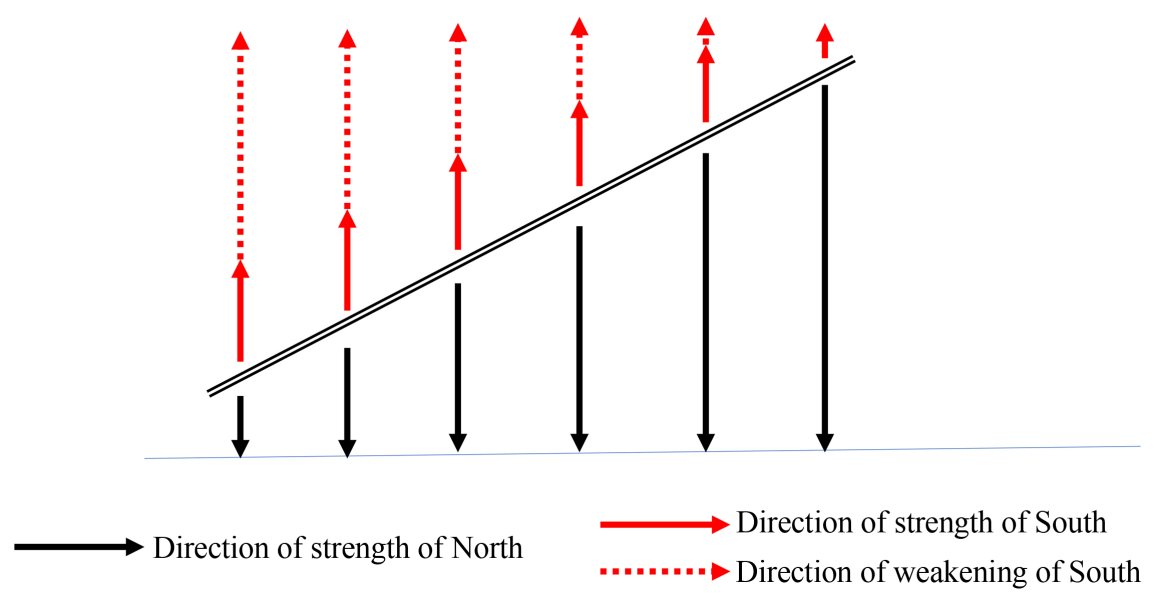

Figure 12. What is happening when two opposite polarizations meet.

Because this zone of annihilation exists, and the phenomenon if it cannot close this vacuum by smashing the magnets together, the magnetic phenomenon quickly loses strength from the north. After this line of annihilation/silence, silence because there is no magnetic activity, everything on this line occupies the same space and returns to what it was before it was a particle, the material that makes up that particle, they cannot occupy the same space.

After this line of annihilation, or quantum magnetic silence we see from Figure 11 and Figure 12 and the data in Appendix 2, that the phenomenon becomes south. Only reaching its peak south strength after the line of silence/annihilation. As depicted in Figure 13.

The south polarized magnetic phenomenon, $K^{s}$ reaches its peak away from the line of silence/annihilation, will be named appropriately when we understand truly what is going on there. It is only then that Equation (1) $M_{p h}=a \mathrm{e}^{-K_{e} P_{e} c}$ takes effect but it needs to be modified to

$$
M_{p h}=M_{a l s} \mathrm{e}^{-K_{e} P_{e} c}
$$

where:

$M_{a l s}=$ Maximum after line of silence.

After this line of silence, before reaching the peak the increasing magnetic phenomenon can be described as:

$$
M_{\text {phals }}=\mathrm{e}^{K_{e} P_{e} c}
$$

where:

$M_{\text {phals }}$ is magnetic phenomenon after line of silence. It starts at zero, that line represents zero magnetic activity and phenomenon wants to avoid meeting the opposite polarization because it means annihilation, and the phenomenon returns to what it is made of.

When leaving the magnet for experiment, phenomenon is north polarized. However, it will become south polarized in direction as all north polarized, $\mathrm{K}^{\mathrm{n}}$ disappears after the line of annihilation. That all phenomenon that is north will 


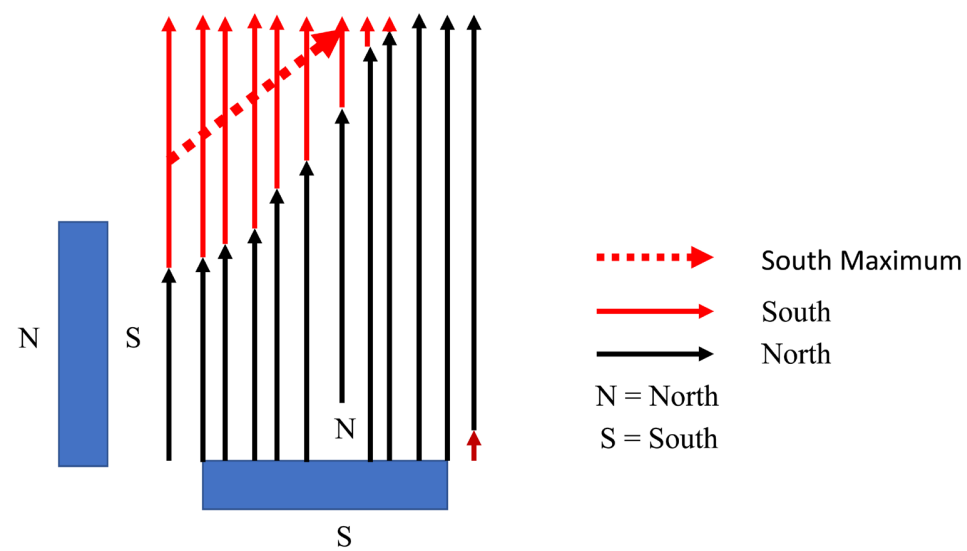

Figure 13. North south magnetic interaction, maximum south.

disappear at the line of silence/annihilation, this means Equation (1) must change to accommodate this new reality given that there is magnetic interaction.

This change is represented by Equation (4)

$$
M_{p h}=\neq a \mathrm{e}^{-K_{e} P_{e} c}-\varnothing
$$

where:

$=$ = determinant of interaction of opposite pole from another magnet and this is determined by

- Strength at source of opposite pole.

- Time it left opposing magnet.

- Angle of interaction, in this case $90^{\circ}$.

- $0<=<1$ The greater the interaction, for example closer the magnet that has the opposite polarized face, the lower the value for the interaction factor.

$\varnothing=$ zerowa $=$ determined by same factors as determinant of interaction. It is equal to the value of the last magnetic phenomenon of the opposite pole that is equal in energy and interacts such that the direction of the phenomenon being measured ceases to exist in that direction.

The zerowa is not constant, it is determined by the time both phenomena left their sources, by both phenomena, it is meant the north polarized, $\mathrm{K}^{\mathrm{n}}$ and the south polarized phenomenon, $\mathrm{K}^{\mathrm{s}}$.

Looking at the data for the second experiment we see that range of Konke for each line that we have enough data to see the south polarized phenomenon start and thus range of where the zerowa would fall as in Figure 1. These values can be found in Table 1 .

Figure 14 shows how distance influences the line of silence and thus the zerowa. There is a base magnet for our readings away from the magnet. If the magnets are similar if they are close together as opposite polarized magnet 1 , then we would get a line of silence 1. This is when the north polarized phenomenon ceases in that direction away from magnet as shown by the arrows and the opposite, south polarized phenomenon becomes north. When the magnet is moved back and becomes opposite polarized magnet 2, we see the line of silence 


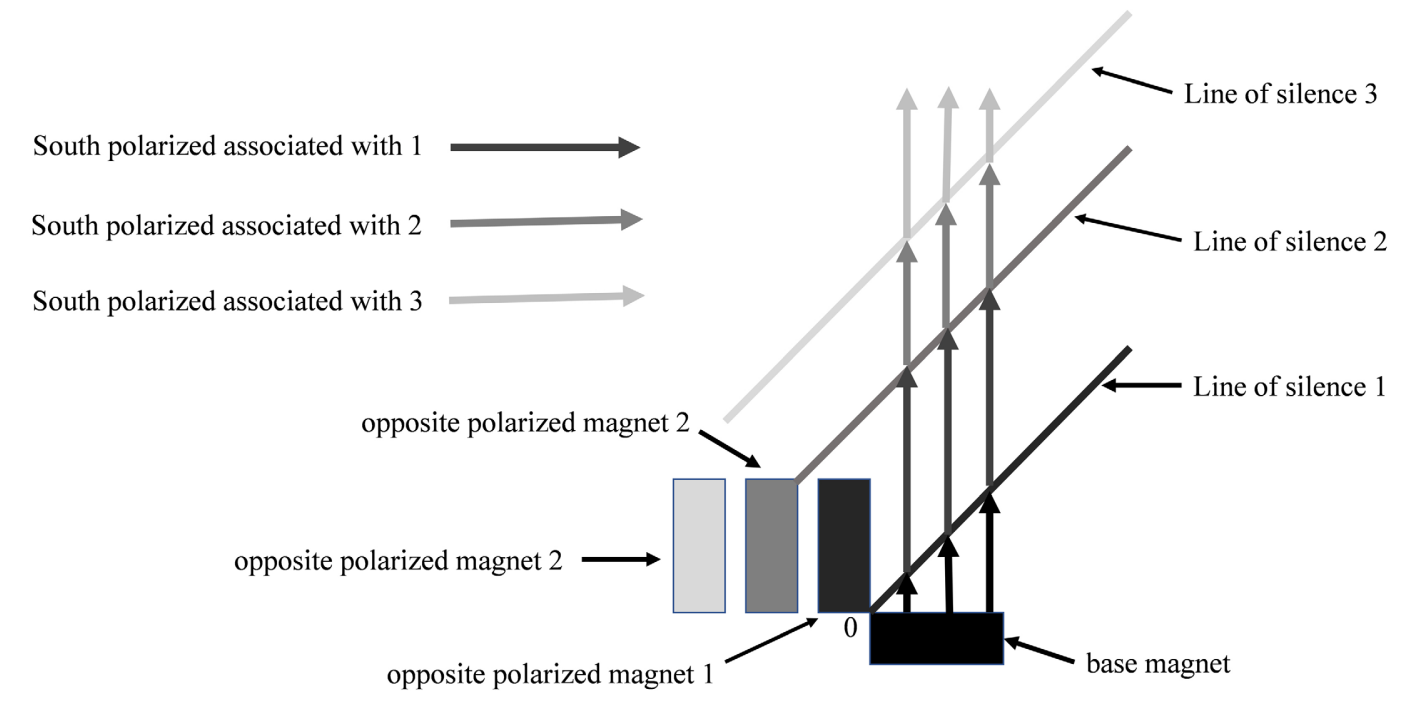

Figure 14. Understanding how distance affects $\varnothing$ and $=$.

Table 1. Approximate value of $\varnothing$.

\begin{tabular}{cccc}
\hline Line & $\begin{array}{c}\text { Approximate } \\
\text { distance of line }\end{array}$ & $\begin{array}{c}\text { Approximate } \\
\text { time to line }\end{array}$ & $\begin{array}{c}\text { Approximate } \\
\text { value of zerowa }\end{array}$ \\
\hline Line 12 & $34<-<35 \mathrm{~mm}$ & $3.4<-<3.5$ jiffies & $0<\emptyset<1.6$ \\
Line 11 & $37<-<38 \mathrm{~mm}$ & $3.7<-<3.8$ jiffies & $0<\emptyset<5.3$ \\
Line 10 & $39<-<41 \mathrm{~mm}$ & $3.9<-<4.1$ jiffies & $0<\varnothing<6.1$ \\
Line 9 & $44<-<45 \mathrm{~mm}$ & $4.4<-<4.5$ jiffies & $0<\varnothing<2.4$ \\
Line 8 & $49<-<50 \mathrm{~mm}$ & $4.9<-<5.0$ jiffies & $0<\varnothing<2.3$ \\
Line 7 & $54<-<55 \mathrm{~mm}$ & $5.4<-<5.5$ jiffies & $0<\varnothing<0.8$ \\
Line 6 & $64<-<65 \mathrm{~mm}$ & $6.4<-<6.5$ jiffies & $0<\varnothing<0.3$ \\
Line 5 & $76<-<77 \mathrm{~mm}$ & $7.6<-<7.7$ jiffies & $0<\emptyset<0.1$ \\
Line 4 & $79<-<80 \mathrm{~mm}$ & $7.9<-<8.0$ jiffies & $0<\emptyset<0.1$ \\
\hline
\end{tabular}

move to position 2, meaning the north polarized phenomenon, $K^{\mathrm{n}}$, remains north in measured direction until line of silence 2 . The same principle when magnet with opposing polarized face is moved back to position 3 .

Figure 14 must be understood, that if magnets change and one emits a much stronger magnetic phenomenon, or the dynamics will change, the relationships will change because of the differences in amount and therefore energy.

The same is true if the angles change, this changes direction of the magnetic phenomenon thus the relationships the different polarized phenomena will have.

The line of silence has evidence of an explosion, when we look at Figure 12 and Figure 13, we can see the phenomenon is affected only reaching its height away from the line of silence when it has become opposite polarized. The magnetic phenomenon is less energized than electrons and much larger particles, but 
that is evidence of a continuous gentle explosion as some of the phenomenon interacts and being polarized in "opposites" there is an explosion causing a "vacuum".

\subsection{Experiment 3}

This experiment involved the set-up set in Figure 15.

The data for this experiment can be found in appendix $\mathrm{C}$ and is graphically represented in Figure 16.

Figures 17(a)-(c) give a closer look at what is happening. We break up Figure 16 to take out the extreme behaviour at the beginning so that we can see a clearer picture for our minds to grasp in smaller components.

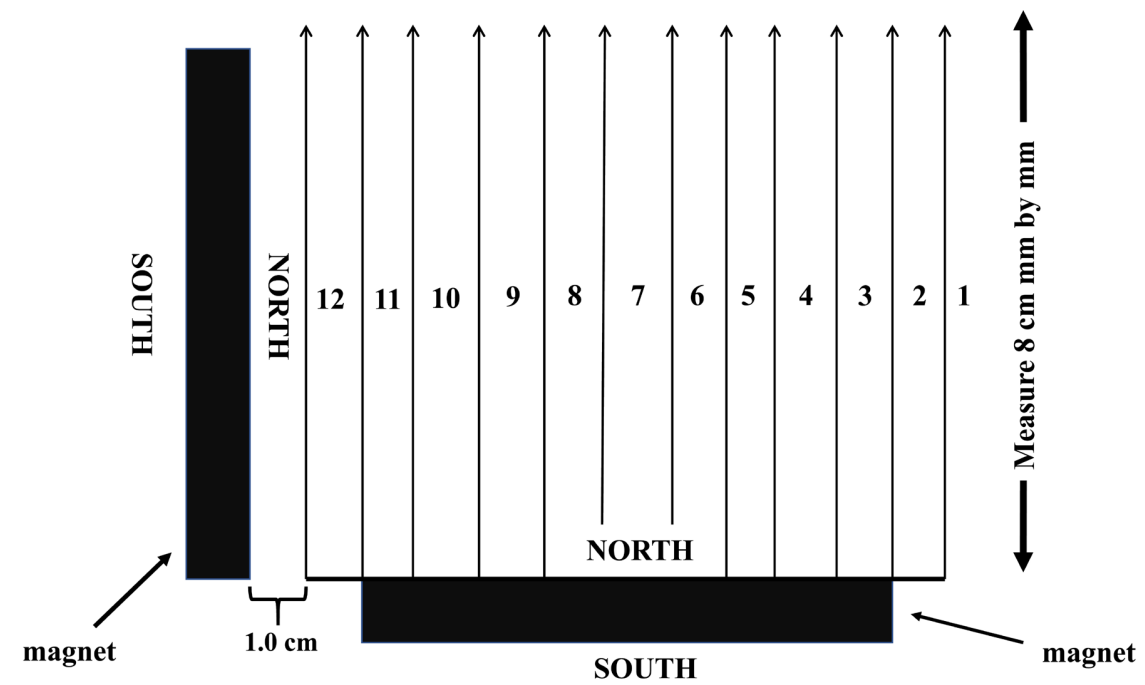

Figure 15. North North $90^{\circ}$ Experiment.

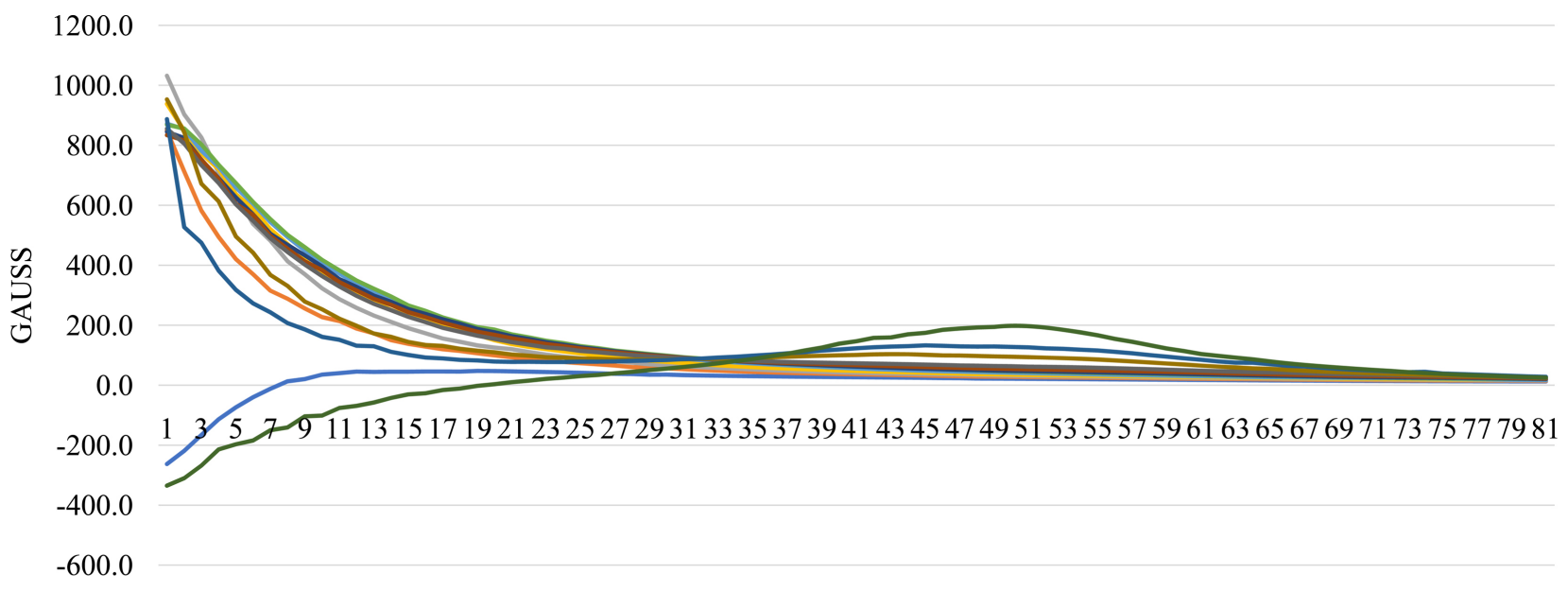

MM

-Line 1 - Line 2 - Line 3 - Line 4 - Line 5 -Line 6

-Line 7 - Line 8 - Line 9 - Line 10 - Line 11 - Line 12

Figure 16. Experiment 3. 


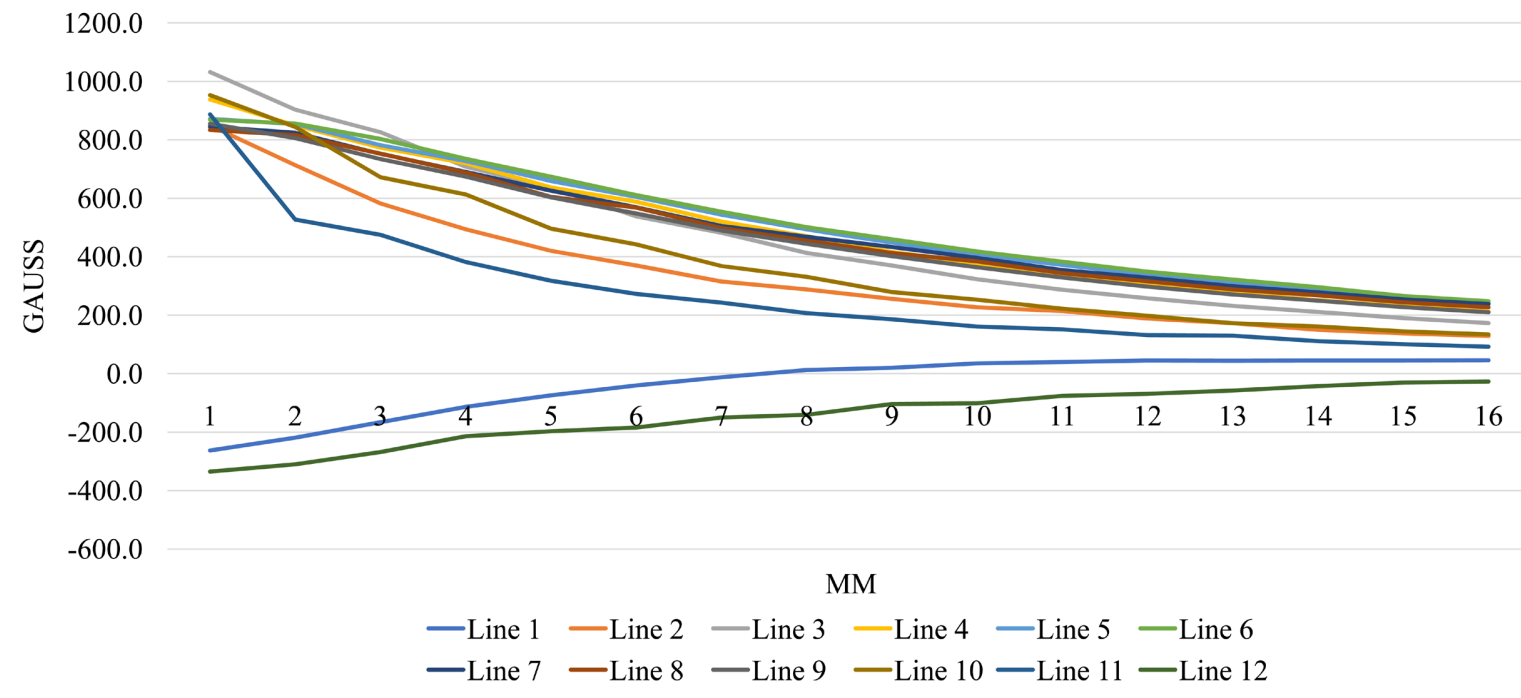

(a)

250.0

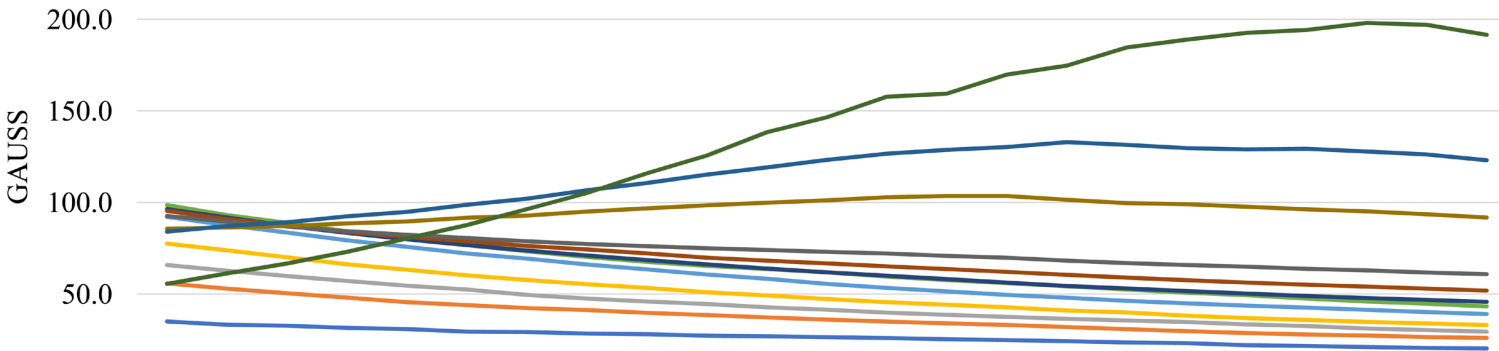

0.0

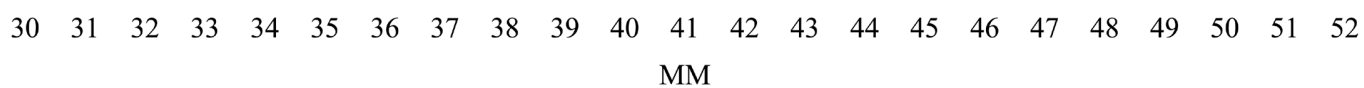

$$
\begin{aligned}
& \text {-Line } 1 \text { - Line } 2 \text { - Line } 3 \text { - Line } 4 \text { - Line } 5 \text { - Line } 6 \\
& \text {-Line } 7 \text { - Line } 8 \text { - Line } 9 \text { - Line } 10 \text { - Line } 11 \text { - Line } 12
\end{aligned}
$$

(b)

250.0

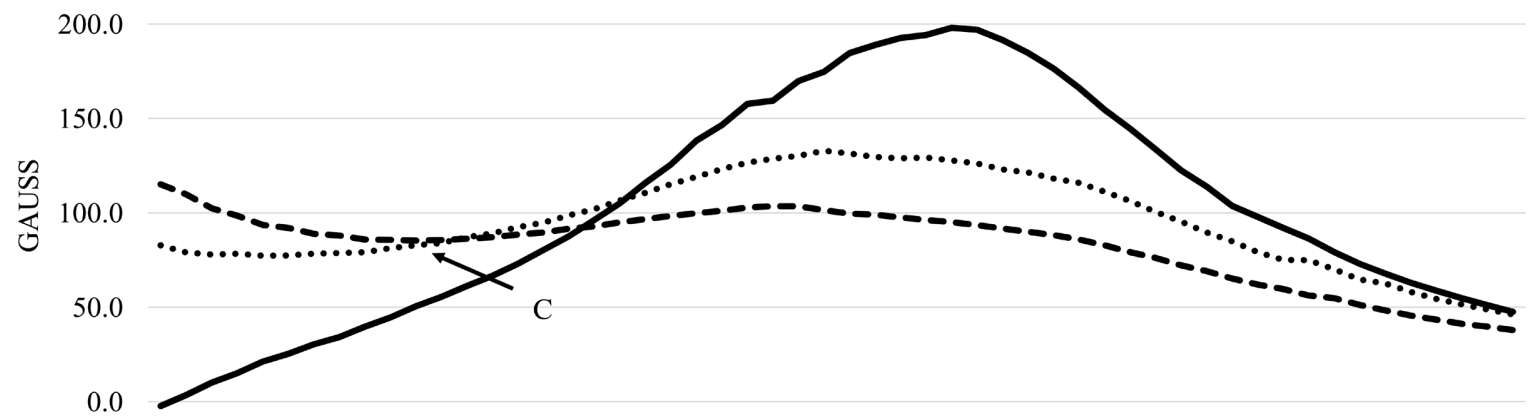

$\begin{array}{lllllllllllllllllllllllllll}19 & 21 & 23 & 25 & 27 & 29 & 31 & 33 & 35 & 37 & 39 & 41 & 43 & 45 & 47 & 49 & 51 & 53 & 55 & 57 & 59 & 61 & 63 & 65 & 67 & 69 & 71\end{array}$ $-50.0$

-- Line $10 \cdots$ Line 11 Line 12

(c) 


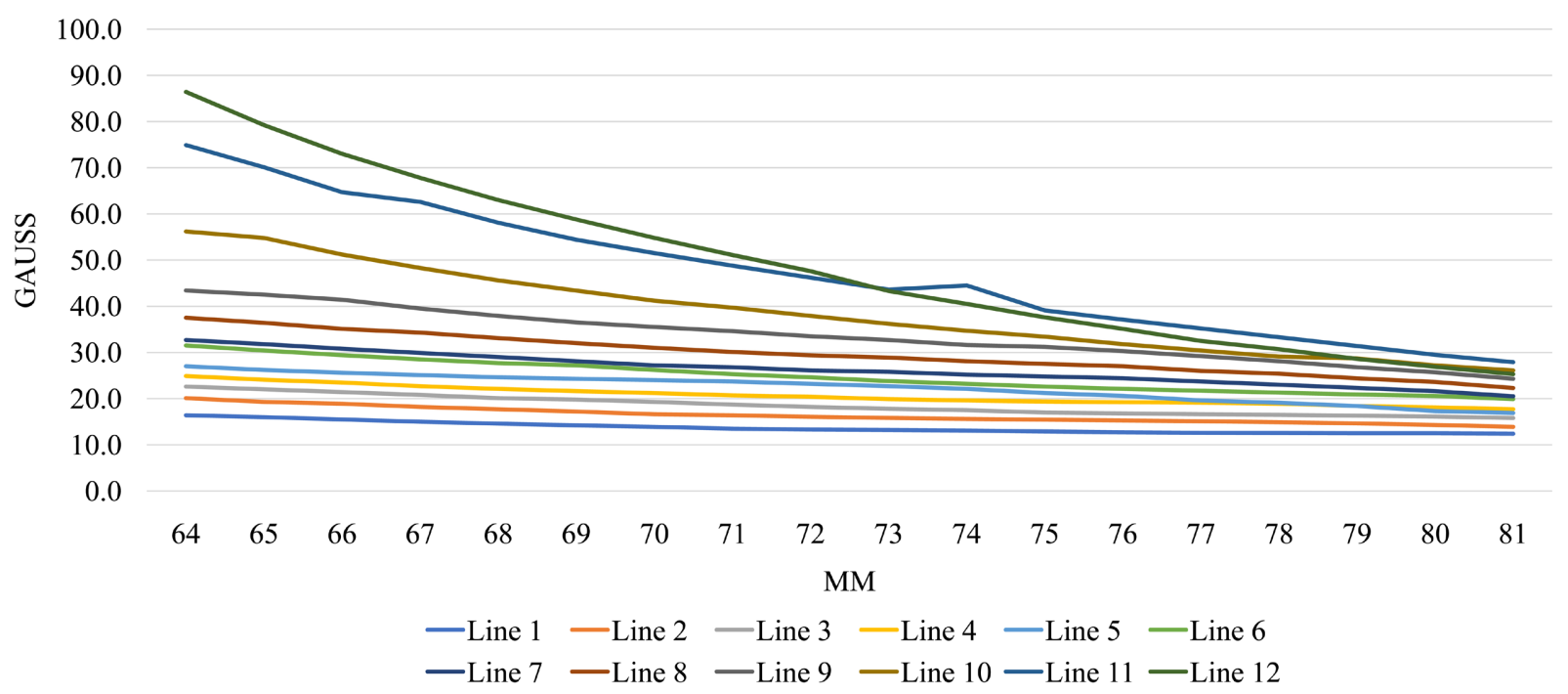

(d)

Figure 17. (a) Experiment 3, 1 - 16; (b) Experiment 3. 30 - 52; (c) Experiment 3, Lines 10 - 12, 19 - 72; (d) Experiment 3,64 - 81.

Figure 17(a) covers the first 1.5 jiffies of this phenomenon from the source. What is interesting is that though in the base experiment lines 1 and 12 both started as south polarized phenomenon. In experiment 2, line 1 started of as south polarized, however line 12 did not. In experiment 3, both line 1 and 12 start of as south polarized as in the base experiment, but line 12 is south for longer and more intense, and rises as a north polarized phenomenon for a more intense period as can be seen in Figure 17(b).

One can see in Figure 17(b) how line 12 rises, but it is not the only line to rise, 11 and 10 also rise, this can only be because of the interaction from similar polarized magnetic phenomenon emanating from the second magnet. We can see this clearly from Figure 17(c).

The clear rise of lines 11 and 12 can easily be appreciated, however for line 10, one needs to look at the data and it rises at measurement 29 , an anomaly, in the base all north phenomenon is falling, this can only be due to the influence of the same polarized magnet, but why and how the rise?

Figure 17(d) shows how the phenomenon ends after 8 jiffies.

\subsubsection{Basic Comparisons}

The first thing that must be appreciated is that after 8 jiffies there is more of this magnetic phenomenon in experiment 3 than in either experiments 1 or 2 in absolute terms. 8 jiffies is how much time it takes for magnetic phenomenon to travel $8 \mathrm{~cm}$, not forgetting in one second it travels approximately 300,000 kilometers.

The reality that there is more of this phenomenon after 8 jiffies, end of our measurements is shown in figures in Figure 18(a) and Figure 18(b). Figure 18(b) being the absolute value, how much of the phenomenon is there be it south or north polarized, whilst Figure 18(a) shows south polarized phenomenon as a negative. 


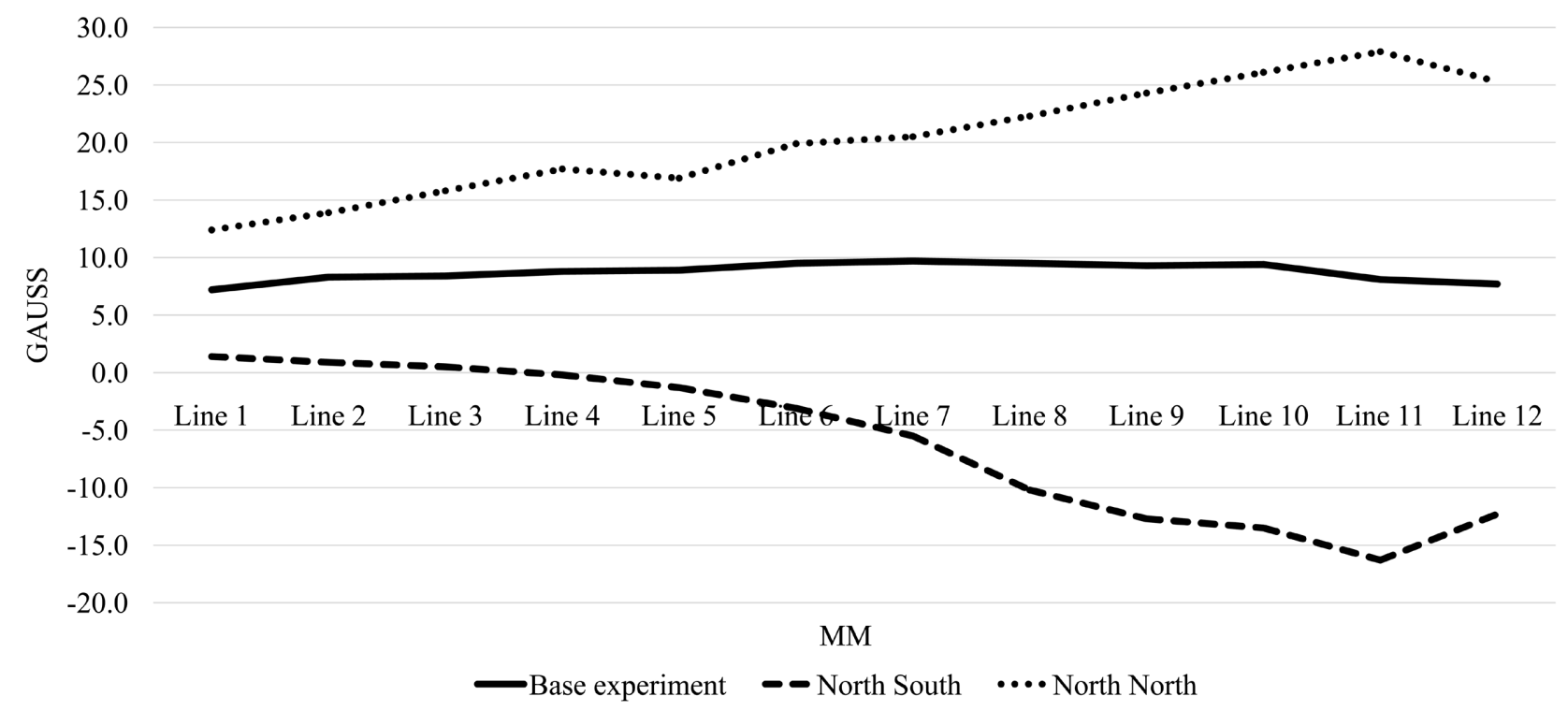

(a)

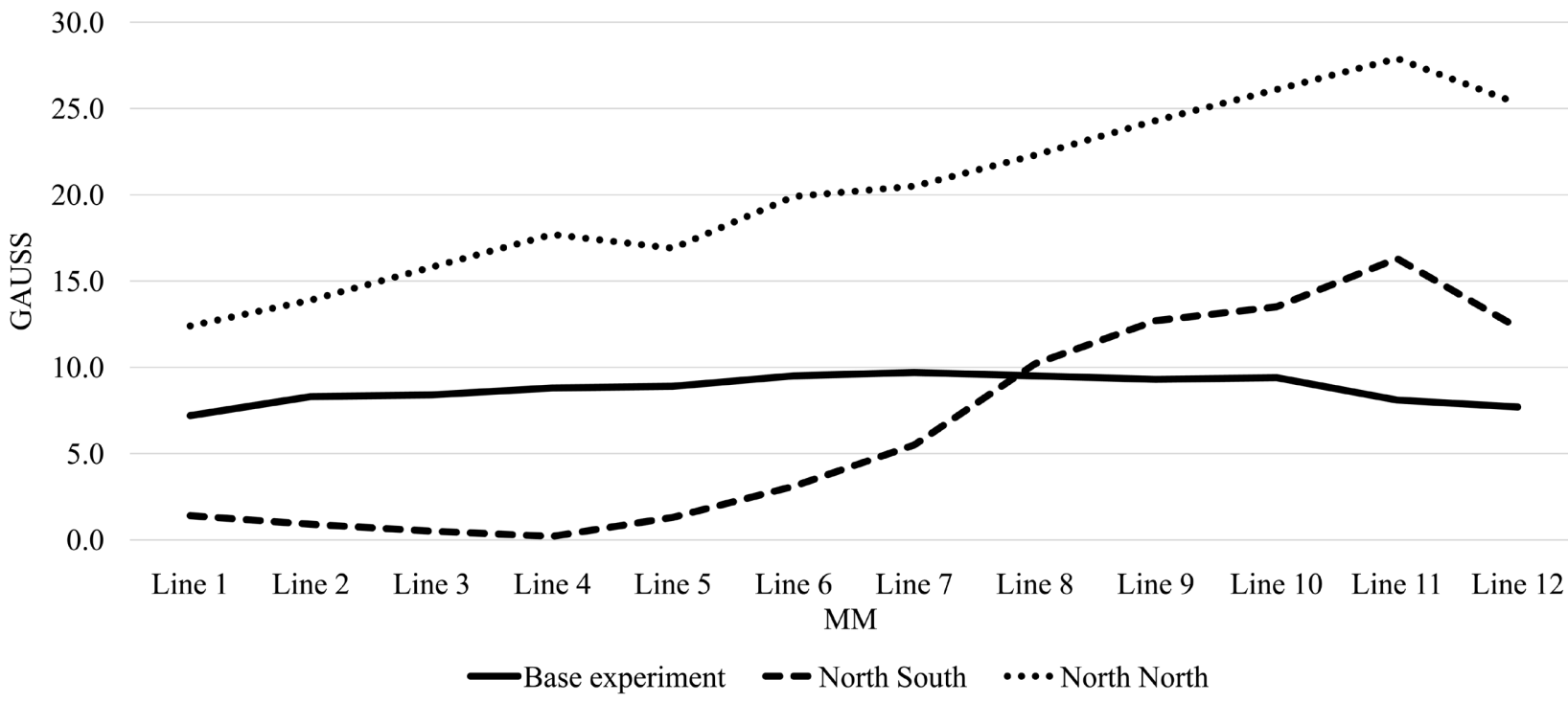

(b)

Figure 18. (a) After 8 Jiffies; (b) After 8 Jiffies absolute values.

As can be seen at the end, only the base experiment has a wave formation as both experiment 2 and 3 have been influenced by the second magnet be they having their face north or south polarized. The second magnet being closer to line 12 will affect this line the greatest because of proximity. In the base experiment there is no other relationship built by the magnetic phenomenon except with that emanating from the same source.

\section{Summation of past history of phenomenon}

Data is there to be meddled with. Looking at Table 2, how much of the phenomenon has existed in each experiment up to 8 jiffies. Understand that the only phenomenon that still exists is the last measurement. But looking at total phenomenon will give us a little view about what has happened, what is happening? 
Table 2. Accumulated past phenomenon.

\begin{tabular}{cccc}
\hline Line & Base Experiment & North South & North North \\
\hline Line 1 & 2714.1 & 3088.7 & 2834.3 \\
Line 2 & 6487.6 & 6700.2 & 8019.7 \\
Line 3 & $11,276.9$ & 9615.0 & $10,589.2$ \\
Line 4 & $11,135.9$ & $10,246.8$ & $11,405.9$ \\
Line 5 & $12,640.2$ & $11,163.0$ & $12,273.9$ \\
Line 6 & $13,178.5$ & $11,837.7$ & $12,700.3$ \\
Line 7 & $13,310.9$ & $11,163.3$ & $12,213.0$ \\
Line 8 & $13,389.6$ & $11,487.0$ & $12,186.7$ \\
Line 9 & $13,155.7$ & $11,929.8$ & $12,130.0$ \\
Line 10 & $12,687.1$ & $11,642.8$ & $11,303.6$ \\
Line 11 & 8384.8 & $12,092.3$ & $10,011.2$ \\
Line 12 & 2809.5 & 9268.5 & 8142.5 \\
Total & $121,170.8$ & $120,235.1$ & $123,810.3$ \\
\hline & & & \\
\hline
\end{tabular}

Given that the Base has no other magnet, a basic quick view is just to look at absolute totals. The Base has had a measured summation of 121,171 gauss, North South 120,235, this is because of the relationship the two opposite polarized phenomena will have at the line of silence/annihilation. Some of the material ceases to exist in the form of magnetic phenomenon as they have a relationship that ends up in them returning to what they are made of.

The north to north relationship has yielded more stuff, but why? It is noted this is not a very accurate measure, but gives an idea, most of this stuff is no longer in existence, destroyed by being unstable on its own, and in the case of north south relationship not only is there the instability of the phenomenon a factor to take into account, but also the line of silence.

Most of the phenomenon is gone after 8 jiffies. Take line 12 from Table 1 with north north relationship, it could be any, but this is about experiment 3 . The total from line 12 for experiment 3 from Table 1 is 8142.5 , that is just a summation, the reality is that after 8 jiffies at line 12 in experiment 3 there is 25.3 gauss, the highest line 12. If one looks at Figure 18(b) one can see the north north relationship measured away from base magnet results in the most gauss at the end, the most phenomenon. Why is this?

\subsubsection{What Is Happening?}

The first thing to understand is that there is more of this magnetic stuff at the end of experiment 3 than either the base experiment or when the experiment involves creating relationships with the opposite polarized phenomenon as in experiment 3 . 
More of this stuff involves having more energy at every level. In the second experiment the relationships established ended up in a line of silence, some of the phenomenon was annihilated, all the north bound phenomenon on the lines being measured gets annihilated at the line of silence, one can understand the reasoning behind Equation (4). These experiments are about what happens between lines 1 and 12. There will be a lot of different relationships taking place beyond line 1, but for these experiments we are not interested, only the changing relationships between 1 and 12 .

As we have more of the stuff annihilation is not taking place, there is no vacuum. As the interacting phenomenon cannot occupy the same space and they are of the same polarization, they push each other away. Newton will help us here, quoting from the NASA website [7], Newtons first law of motion states "every object will remain at rest or in uniform motion in a straight line unless compelled to change its state by the action of an external force."

We most clearly see this with lines 12,11 , and 10 in experiment 3 . The resultant relationships created by the base magnet with the second magnet of same polarization have resulted in more of the magnetic phenomenon, even increasing defying the downward trend especially when it comes to lines 11 and 10.

Unable to occupy the same space the phenomenon pushes each other away. To understand this, graphic representations might be of most useful help. This is why Figures 19(a)-(e) are most helpful.

Figure 19(a) is a pictorial illustration, and only that to help us understand. It shows magnetic phenomenon coming out of the base magnet, this phenomenon is north polarized, $\mathrm{K}^{\mathrm{n}}$. Figure 19(a) is very simplified, but as one can visually see, closer to the source, region $\mathrm{A}$, that the magnet phenomenon is tightly packed. It is less packed in region $B$. This is understandable because we know the phenomenon is unstable and declines rapidly, the reasoning behind Equation (1). In region $C$ it is most sparse, not as intense as region $B$ which is less intense than region $\mathrm{A}$.

Figures 19(b)-(d) show the north polarized phenomenon from the base magnet interacting with the north polarized phenomenon. Figure 19(e) will not be discussed as it involves experiment 2, but one gets the idea.

In Figure 19(b) it can be seen that the source of both phenomena is close as possible, we can see how they interact visually. The further one moves away from the base magnet close to the opposing magnet, there will be a stage when magnetic phenomenon from the base magnet is not as strong as magnetic phenomenon from secondary magnet close to the secondary magnet. This means the magnetic phenomenon from the base magnet is not as strong as the magnetic phenomenon from the second magnet. This explains why lines 12,11 , and 10 we see a rise.

The rise is because any similar phenomenon can never occupy the same space, they push each other, if they where opposite polarized annihilation. This push is clearly seen, as lines 12,11 , and 10 are closest to the opposing magnet in experiment 3, we see this effect with them rising thus nullifying Equation (1). 


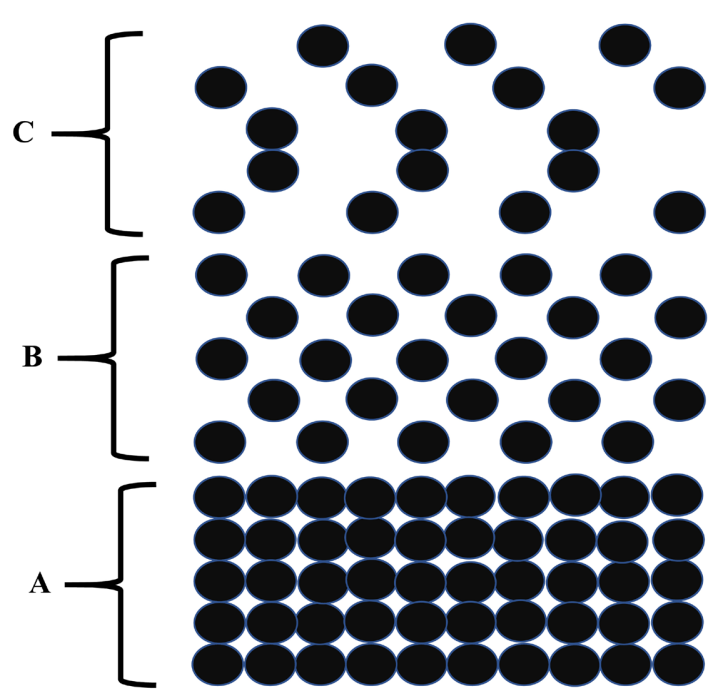

$\mathrm{K}^{\mathrm{n}}$ magnetic phenomenon

(a)

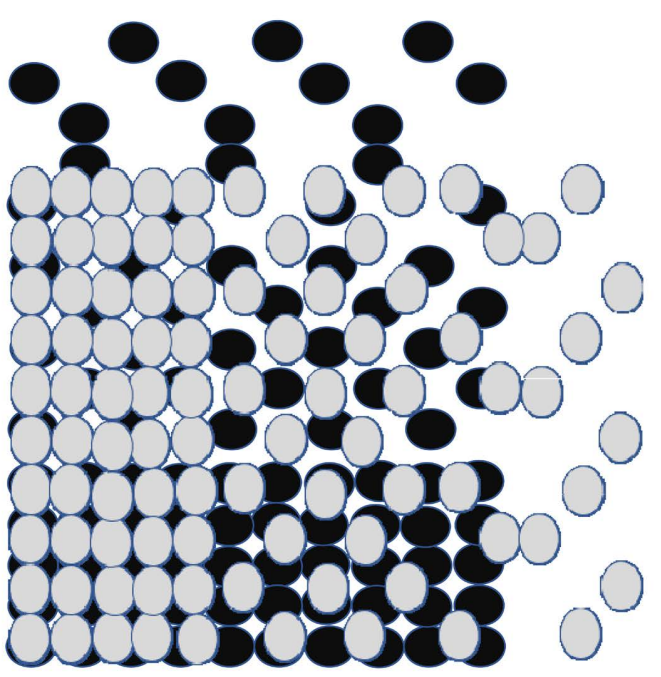

(b)

$\mathrm{K}^{\mathrm{N}}$ magnetic phenomenon from base magnet

$\mathrm{K}^{\mathrm{N}}$ magnetic phenomenon from second magnet

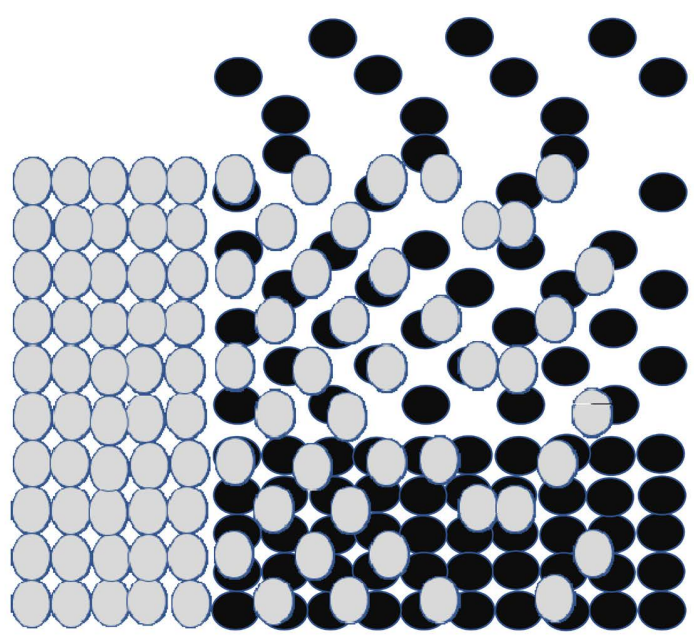

(c) 
$\mathrm{K}^{\mathrm{N}}$ magnetic phenomenon from base magnet

$\mathrm{K}^{\mathrm{N}}$ magnetic phenomenon from second magnet

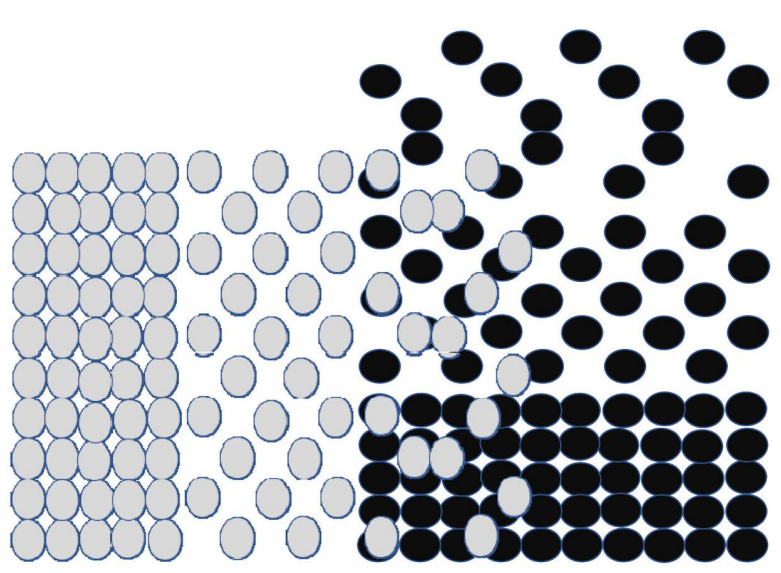

(d)

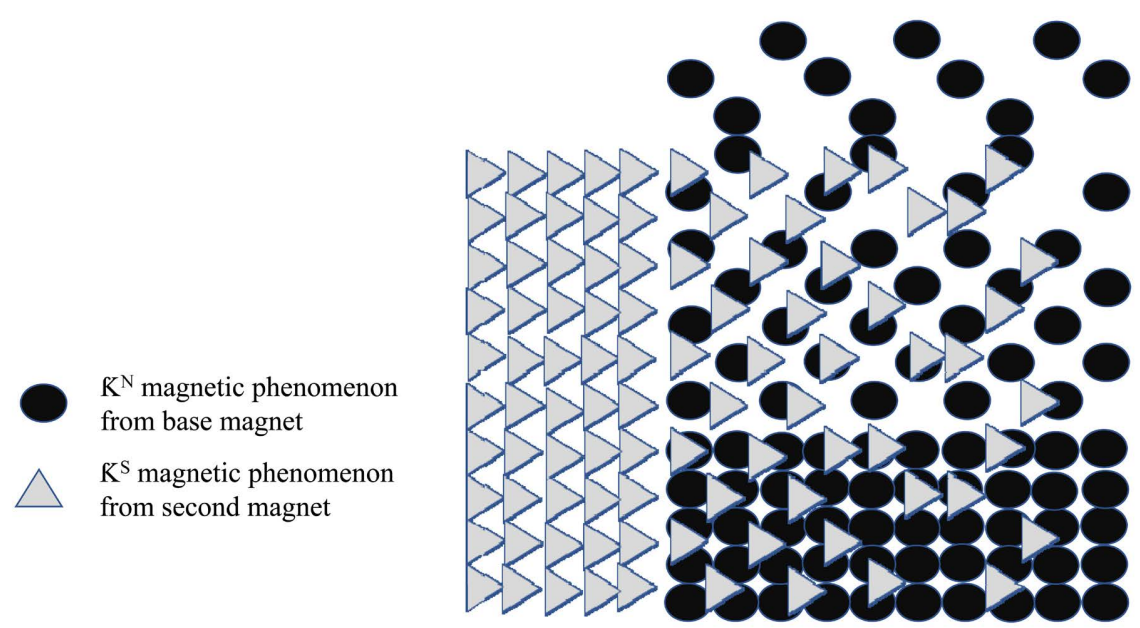

(e)

Figure 19. (a) Magnetic phenomenon from base magnet; (b) Magnetic phenomenon from base magnet interacting with phenomenon from secondary magnet; (c) Magnetic phenomenon from base magnet interacting with phenomenon from secondary magnet; (d) Magnetic phenomenon from base magnet interacting with phenomenon from secondary magnet; (e) Magnetic phenomenon from base magnet interacting with opposite polarized phenomenon.

However, one can see as we move the secondary source back that the relationship between the phenomenon from the 2 sources changes. When we move the phenomenon back the secondary magnet source gets weaker and weaker as can be seen from the illustrations reducing the chances of the material "bumping" into each other.

When this phenomenon bumps into each other it must be remembered it is not stable. But we know that a lot of the material is bumped up because of the way the magnets are set up, we can see that after 8 jiffies experiment 3 has the most magnetic phenomenon be it south polarized or north polarized.

We can see why from the illustrations, understanding if we had moved the magnet closer we would get a greater effect.

When one thinks they are holding a pole, they are not really touching it, simi- 
lar phenomenon will not occupy the same space, even if one smashes a fly with a sledgehammer, they don't break the atomic composition, even a sledgehammer does not allow one to break the forces that hold an atom together.

The same principles apply with the magnetic phenomenon, it is the laws of Isaac Newton, they push each other. It was a very premature rush to cancel Isaac Newton out of quantum mechanics without understanding the simplicity of things.

Take Isaac Newton's [6] third law, "for every action (force) in nature there is an equal and opposite reaction. In other words, if object A exerts a force on object $\mathrm{B}$, then object $\mathrm{B}$ also exerts an equal force on object A."

When the base magnetic phenomenon meets with magnetic phenomenon from the secondary magnet, they both exert a force on each other pushing each other in different directions, the degree of push depending on the intensity of probability phenomenon will meet, the magnets being closer means greater degree of push as there is more stuff.

It does not matter how the push occurs, it occurs, and that is from Isaac Newton. One of cause can argue that perhaps newtons laws have everything to do with magnetic phenomenon that is similar, but what about when south meets north and the line of silence. However, Newton's principles apply to everything because everything is in a relationship.

The [8] second law of everything which derives from Newton's first law states that "an information package will remain in its present state unless a relationship is established." When the phenomenon from base magnet meets any other similar phenomenon be it south or north a relationship is established.

When similar polarized magnetic phenomenon gets into a relationship as in experiment 3 Equation (1) and Equation (5).

$$
M_{p h}=\models a \mathrm{e}^{-K_{e} P_{e} c}
$$

where

$\models=$ determinant of interaction of similar polarized phenomenon from another magnet and this is determined by

- Strength at source of opposite pole

- Time it left opposing magnet

- Angle of interaction, in this case $90^{\circ}$

- $\geq 1$

\section{Further Analysis}

Both known laws of everything are at play and obvious with the magnet. Everything is in a relationship, there is nothing that can exist outside of a relationship. This being so, it means laws about relationships are about everything. The first known laws that apply to everything are:

- Every relationship has a loss of freedom due to the energy needed to maintain them.

- An information package will remain in its present state unless a relationship 
is established.

Take the three experiments that have been discussed for this paper. In experiment 1 , the base experiment we measure away from the magnetic face. The magnetic phenomenon is in a relationship by itself, for us to merely identify it means it has a different structure than other phenomena. It is energy that gives it a structure.

When we introduce a secondary magnet the [9] second law of everything applies. An information package will remain in its present state unless a relationship is established, be the secondary magnet emitting phenomenon with the same polarization or different. A relationship is established, and we see this with the different results, and the principles implied. Establishing relationships because all relationships take energy to maintain means that new energy balances are implicated within the confines of the magnetic phenomenon.

\section{Comparing the Three Experiments}

The most obvious difference is that the base experiment ends with a classic wave pattern in the first 8 jiffies, however both experiment 2 and 3 have more of the phenomenon in lines 12,11,10,9, there is no classic wave pattern. Though not classical waves, Schrodinger's equation limited by the different equations for each experiment should still suffice.

Take Figure 20(a). It shows the magnetic behaviour in base experiments. We can see through interactions that on both sides we have a little south polarized phenomenon at lines 1 and 12. These areas are shown as A and B.

The second experiment is shown in Figure 20(b).

We understand that there is a line of silence in the relationships established, by occupying the same space, the phenomenon destroys each other and breaks into what makes the magnet. What is interesting is that at B there is south polarized phenomenon but at $\mathrm{A}$ there is none of this. The reason for this is that in experiment 2 we have the south polarized face of the second magnet emitting south polarized magnetic phenomenon. This phenomenon released by the second phenomenon will be north polarized at A and it annihilates itself, we know opposite polarized phenomenon annihilate each other.

Figure 20(c) illustrates the third experiment.

Experiment three is looking at north north magnetic phenomenon interaction. We see that at $B$ we have south polarized phenomenon as in all the other experiments, we also have it at A but it is more intense. The reason for this is that at $\mathrm{A}$, south polarized phenomenon is associated with both magnets. As they are similar polarized, they push each other, more evidence of this reality and we see a greater intensity of south polarized phenomenon in line 12 .

\section{Magnetism and Computing}

Quantum phenomenon all has its unique characteristics. However, with thought and further investigation they can all be used in the similar ways when it comes 


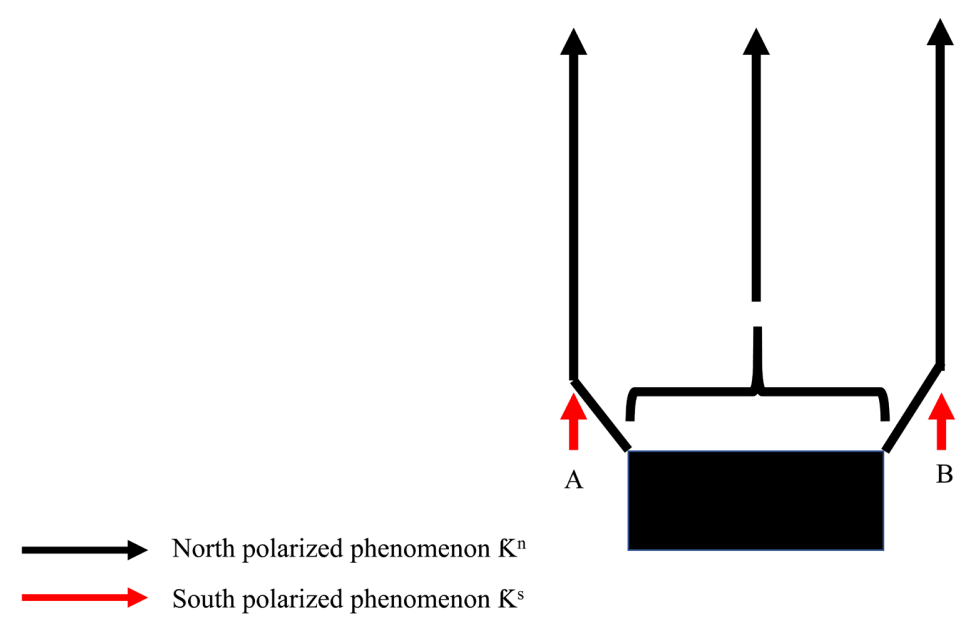

(a)

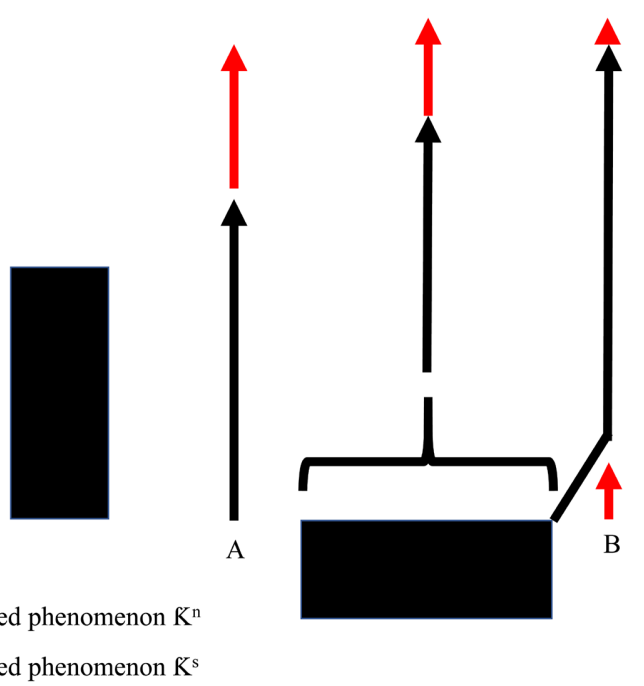

(b)

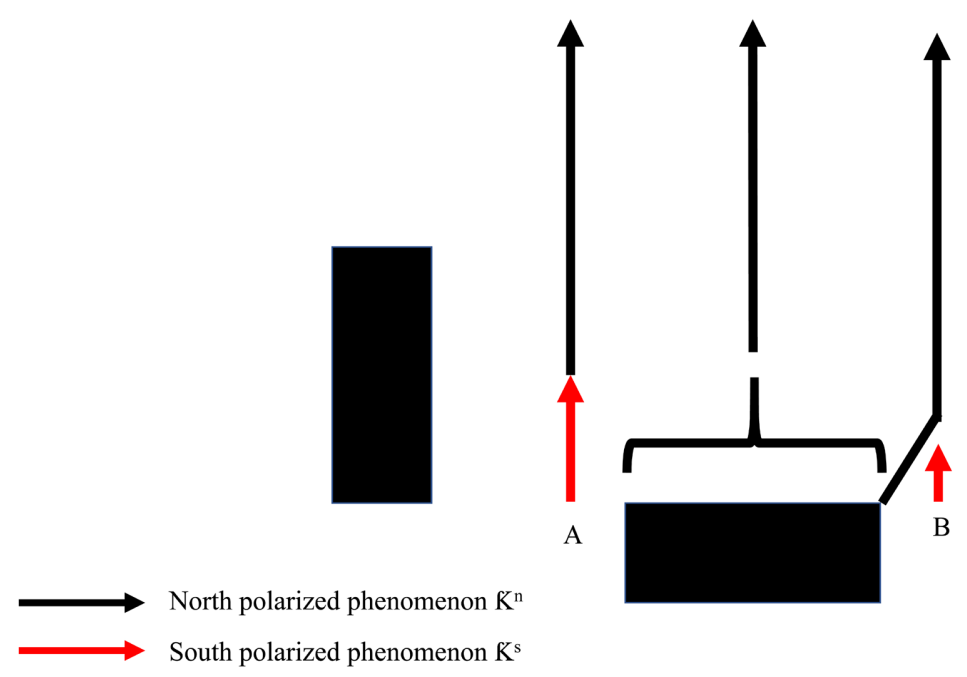

(c)

Figure 20. (a) Behaviour of magnetic phenomenon experiment 1; (b) Behaviour of magnetic phenomenon experiment 2; (c) Behaviour of magnetic phenomenon experiment 3. 
to computation. There is already theoretical talk of these properties being useful in quantum computing. "In the spin-chain model, we can extend the communication model from the single qubit as initially defined to a collection of flying qubits that transmit a certain amount of information... For instance, if in the exchange interaction, we take the coupling constant such that $J<0$, we get the case of the ferromagnets... [10]" further theoretical calculations are made. This thought can only come from accepting reality that magnetism and its properties can be utilized to calculate. Only something discrete can be used to calculate.

The original paper showed that a [11] monopole can be created experimentally, meaning in this world not theoretically. What seems simple engineering, we create, instead of using a permanent magnet to create the monopole, use an electrical magnet, then we can be assured at the microchip level at the least, that we can control the strength of the pulse of the magnetic wave, thus fully understanding magnetic phenomenon first from a binary level than with further investigation utilizing it in quantum information processors. The different electrical power being tuned to get a definite one or zero from magnetic particles created.

\section{Conclusions}

Though not an original aim of these experiments and hence this paper, experiment 1 shows the chaos of the phenomenon in acceptable detail as it shows the chaos before the wave formation. Given the potential amount of calculations needed to get to a wave formation, in less than 2 jiffies of a second, even $0.1 \%$ in practice would be staggering compared to anything that exists or potentially has been thought of, that is the original algorithm, it tells us if this happens in such proportions, this will be the result, and this is usually expressed mathematically, at the most basic dealing with energy.

It can only organize itself into a wave formation in such a short time, jiffies are very small time frames, because it is going through a medium, why bother? This proof because of experiment 1 , is irreprovable, it is something that organizes itself into a wave formation, that something is a particle associated with magnetism that is aptly called a khumalon. The detail cannot be ignored.

Understanding that the phenomenon is particles that organize themselves into waves, it means what one is studying when looking at magnetic phenomenon in relationships, one has a very cheap but effective particle collider. This particle collider allows us to see, with a low energy phenomenon, it is a "cooling" system for particles, versus a photon, a "regulation" system for atoms. Atoms are made up of several particles.

This particle collider involves an already unstable phenomenon, but it allows us to see what is happening when particles collide, and one can then add more and more energy for larger phenomenon colliding.

With no doubt opposite polarized phenomenon there is annihilation involved as predicted and proven that phenomenon of the opposite polarization ends up 
in annihilation, particle, and anti particle. South is the anti particle of north.

With same polarization, there is no annihilation but a push. Same as what happens when all other particles meet their anti-particle, annihilation.

This line of annihilation is something that obviously needs further investigations; this is where we see in a gentle manner particle annihilating each other, returning to what they are made off. It must have potential for our use, with time with more precision, perhaps firing electrons or photons through there and observing will help us a lot with understanding the nature of what is going on. It is promising for something.

The phenomenon behaves and follows the laws of relationships, the laws of everything as everything is in a relationship, we see it changing behaviour as new relationships, or different relationships are created, different interactions of energy. Newton's ideas inspired the second law.

\section{Conflicts of Interest}

The author declares no conflicts of interest regarding the publication of this paper.

\section{References}

[1] Khumalo, B. (2021) Magnetism: Insights from the Thomas Young Experiment. Journal of Advances in Physics, 19, 185-203. https://doi.org/10.24297/jap.v19i.9090

[2] Khumalo, B. (n.d.) Magnetism: Further Proof of Wave Particle Duality. (Not Yet Published)

[3] Khumalo, B. (2021) The Algorithm of Information and the Origin of Basic Particles. Journal of Advances in Physics, 16, 479-497. https://doi.org/10.24297/jap.v16i1.8485

[4] NASA (2020) The Magnetosphere Our Shield in Space. https://history.nasa.gov/EP-177/ch3-4.html

[5] Elert, G. (2002) Speed of Light: The Physics Factbook. https://hypertextbook.com/facts/2002/JohnnyAlicea2.shtml

[6] Boldyreva, L.B. (2019) Spin Supercurrent in Phenomena of Quantum Non-Locality (Quantum Correlations, Magnetic Vector Potential) and in Near-Field Antenna Effect. Journal of Modern Physics, 10, 128-144. https://doi.org/10.4236/jmp.2019.102010

[7] NASA (2020) Newton's Laws of Motion. https://www1.grc.nasa.gov/beginners-guide-to-aeronautics/newtons-laws-of-motio n/

[8] Unit Converters (2008) Convert Planck Length to Centimeter. https://www.unitconverters.net/length/planck-length-to-centimeter.htm

[9] Khumalo, B. (2016) The Theory of Everything: Newton, Information, Complexity, and Evolution.

https://instituteofknowledge.blogspot.com/2016/11/the-theory-of-everything-newto $\underline{\text { n.html }}$

[10] Majumdar, M.G (2018) Quantum Information Processing Using the Exchange Interaction. Journal of Quantum Information Science, 8, 139-160. 
https://doi.org/10.4236/jqis.2018.84010

[11] Khumalo, B. (2021) Longest Monopole.

https://www.youtube.com/watch?v=lthiimcEZVk\&t=210s 